\title{
Experimental calibration of a Cahn-Hilliard phase-field model for phase transformations in Li-Sn electrodes
}

\author{
Srivatsan Hulikal ${ }^{* 1}$, Chun-Hao Chen ${ }^{\dagger 1}$, Eric Chason ${ }^{\ddagger 1}$, and Allan Bower ${ }^{\S}$ \\ ${ }^{1}$ School of Engineering, Brown University, Providence, RI 02912
}

\begin{abstract}
Experiments, numerical simulations, and analytical calculations are used to calibrate a diffuse-interface Cahn-Hilliard model of Li-Sn thin film electrodes that experience a transformation from $\mathrm{Sn}$ to $\mathrm{Li}_{2} \mathrm{Sn}_{5}$. It is shown that a concentration-gradient dependent mobility can be used in the Cahn-Hilliard equation to give the interface a finite mobility and capture its nonequilibrium behavior. Comparing experiments and simulations, the free-energy of Li-Sn, diffusivity of $\mathrm{Li}$ in $\mathrm{Sn}$ and $\mathrm{Li}_{2} \mathrm{Sn}_{5}$, the exchange current density for the surface reaction, and the mobility of the $\mathrm{Sn} / \mathrm{Li}_{2} \mathrm{Sn}_{5}$ interface are extracted. The implications of finite interface mobility for practical battery electrodes are discussed.
\end{abstract}

\section{Introduction}

Diffusional phase transformations play an important role in many Li-ion battery electrodes. Cathode materials such as $\mathrm{LiFePO}_{4}, \mathrm{Li}_{x} \mathrm{CoO}_{2}$, and $\mathrm{Li}_{x} \mathrm{MnO}_{4}$ [1], and anodes such as graphite, silicon [2,3], and tin (Sn) [4] undergo phase transformations upon lithiation and delithiation. The charge-discharge dynamics of a battery depends critically on the nucleation and propagation of the phases. In some materials, stresses developed due to volume change during phase transformation and mismatch strain across the phase boundary are significant and affect transport properties $[5,6]$. The stresses can also lead to the degradation and failure of the electrode thus affecting the life of the battery $[7,8]$. Interface propagation is accompanied by energy dissipation which limits the efficiency [9]. Thus, a good understanding of these phase transformations using experiments and modeling is imperative in designing better batteries.

Phase-field models have been used widely in studying phase transformation phenomena [10-16]. In these models [10,11], a continuous field variable represents the phase of the material: for example, in the Cahn-Hilliard model [17], the concentration of the diffusing species is the phase variable. The free-energy is assumed to be a non-convex function of the concentration, so that the system naturally phase-separates. A contribution to the free-energy from the concentration gradient results in the formation of a diffuse phase-boundary between phases. Phase-field models have several advantages. Since the phase variable is continuous, explicit interface tracking is unnecessary. Their variational nature aids the development of stable numerical methods. In addition, Cahn-Hilliard models can also nucleate new phases.

*srivatsan_hulikal@brown.edu

†chun-hao_chen@brown.edu

†eric_chason_phd@brown.edu@brown.edu

§Allan_Bower@brown.edu @brown.edu 
Cahn-Hilliard was first used to study battery materials by Han et al. [12] who determined the diffusivity of $\mathrm{Li}$ in Olivine $\mathrm{LiFePO}_{4}$ using Galvanostatic Intermittent Titration Technique (GITT) and Potentiostatic Intermittent Titration Technique (PITT) experiments and evaluated the effect of gradient energy on transport. Since then, it has been used widely to study several aspects of phase separation phenomena. Using the Cahn-Hilliard model with new insertion kinetics for the surface-reaction, Singh et al. [15] studied the effect of anisotropic mobility on the intercalation dynamics and found a surface-reaction-limited regime where phase-boundaries move along the electrode surface as opposed to the shirking-core models where they move within the bulk. Burch/Bazant [14] showed that the spinodal and miscibility gaps become smaller with decreasing particle size leading to phase-separation suppression in nanoparticles. Bai et al. [18] used an averaged phase-field model and showed the disappearance of spinodal resulting in homogeneous lithiation of nanoscale $\mathrm{LiFePO}_{4}$ particles which may explain their high-rate behaviour. The effect of stress has also been included, for example Cogswell/Bazant [13] studied how coherency strain influences phase-boundary orientation and microstructure morphology in $\mathrm{LiFePO}_{4}$. Coupling a phase-field model with elasticity, Tang et al. [19] looked at the effect of particle size, misfit strain, and electric overpotential in the amorphization of nanoscale olivines. Based on a "microforce balance" approach, Anand [16] extended the Cahn-Hilliard model to account for large elastic-plastic deformations. Using this, Di Leo et al. [20] studied the effect of plasticity on state-of-charge and energy dissipation in amorphous Si electrodes. Apart from single electrode particles, phase-field models have also been used to study porous electrodes [21].

Cahn-Hilliard models contain a number of material parameters that must be determined experimentally. In this paper, our goal is to calibrate a Cahn-Hilliard model of Li-Sn by careful comparison of predictions with experiments. We use the Li-Sn system as a representative example of a practical battery electrode material that experiences several phase transformations during $\mathrm{Li}$ insertion and removal. Sn, with a large theoretical gravimetric capacity of about 990 $\mathrm{mAh} \mathrm{g}^{-1}$ is a promising anode material for Li-ion batteries. Li-Sn serves as a canonical system because upon lithiation, it undergoes a series of crystalline-crystalline phase transformations that are reversible [4,22-25]. Wen/Huggins [22] showed that the Li-Sn system has six phases at high temperature: $\mathrm{LiSn}, \mathrm{Li}_{7} \mathrm{Sn}_{3}, \mathrm{Li}_{5} \mathrm{Sn}_{2}, \mathrm{Li}_{13} \mathrm{Sn}_{5}, \mathrm{Li}_{7} \mathrm{Sn}_{2}$, and $\mathrm{Li}_{22} \mathrm{Sn}_{5}$. Using in-situ XRD, Rhodes et al. [4] identified phases $\mathrm{Li}_{2} \mathrm{Sn}_{5}, \mathrm{LiSn}$, and $\mathrm{Li}_{22} \mathrm{Sn}_{5}$ during lithiation cycles at room temperature. Many studies have looked at the equilibrium properties of Li-Sn and phase diagrams have been constructed (see [23-25] and references therein). For example, plateau potentials of the successive phase transformations at $25^{\circ} \mathrm{C}$ range from $0.76 \mathrm{~V}$ to $0.38 \mathrm{~V}$ (against $\mathrm{Li} / \mathrm{Li}^{+}$) [25]. Apart from equilibrium properties, kinetic parameters have also been measured. The chemical diffusivity of $\mathrm{Li}$ in the various phases at $415{ }^{\circ} \mathrm{C}$ is of the order of $10^{-5} \mathrm{~cm}^{2} \mathrm{sec}^{-1}$ [26]. At room temperature, diffusivities of $10^{-8}-10^{-7} \mathrm{~cm}^{2} \mathrm{sec}^{-1}$ for $\mathrm{Li}_{0.7} \mathrm{Sn}$ and $\mathrm{Li}_{2.33} \mathrm{Sn}$ have been reported [27]. Much smaller diffusivities $\left(10^{-16}-10^{-14} \mathrm{~cm}^{2} \mathrm{sec}^{-1}\right)$ have been observed for Sn $[28]$.

Here, we focus on transformations between $\mathrm{Sn}$ and $\mathrm{Li}_{2} \mathrm{Sn}_{5}$ which are the first two phases to form at room temperature. We measure the in-situ variation of current and voltage in Sn thin film electrodes with Li counter-electrode subjected to lithiation and delithiation (Section 2). Comparing predictions of a Cahn-Hilliard model with experimental observations, we determine the free-energy of $\mathrm{Li}-\mathrm{Sn}$ as a function of $\mathrm{Li}$ concentration, the diffusivity of $\mathrm{Li}$ in the first two phases ( $\mathrm{Sn}$ and $\mathrm{Li}_{2} \mathrm{Sn}_{5}$ ), the surface-reaction parameters in the Butler-Volmer equations, and most importantly, the interface mobility (Section 6). As far as we know, these are the first measurements of diffusivity in $\mathrm{Li}_{2} \mathrm{Sn}_{5}$, the surface-reaction rate for the insertion reaction at the electrode/electrolyte interface, and mobility of the $\mathrm{Sn}_{-} \mathrm{Li}_{2} \mathrm{Sn}_{5}$ interface. The standard Cahn-Hilliard equations predict that the interface has infinite mobility thus ignoring the nonequilibrium interface behaviour which can be important in nanoscale electrodes. Fol- 
lowing Langer and Sekerka [29], we show that the Cahn-Hilliard equations can be modified to model interface-limited processes by including a concentration-gradient dependent mobility of Li (Section 3). We show representative examples that elucidate the general behavior of the modified Cahn-Hilliard model (Section 5).

To better understand interface behavior, we find it helpful to study the sharp-interface limit of the Cahn-Hilliard equations. Accordingly, we present results of a perturbation analysis of the modified Cahn-Hilliard equations based on a paper by Langer and Sekerka [29]. We derive a general energy-based sharp-interface model that identifies the fluxes and conjugate forces for dissipative processes in the bulk and at the interface. A comparison with the perturbation analysis results reveals that the kinetic relations for the interface implied by the Cahn-Hilliard equations are a particular special case of a more general class (Section 4). We discuss the implications of interface mobility for the charge/discharge dynamics and energy efficiency of a battery (Section 7).

\section{Experiment}

We briefly review the experimental procedure used in this study. The apparatus consists of a Li-ion half-cell with a thin-film Sn working electrode and a Li metal foil counter-electrode as illustrated in Figure 1. The Sn thin film was deposited on silica quartz wafers $(50.8 \mathrm{~mm}$ diameter, $450-500 \mu \mathrm{m}$ thick, double-sided polished). Prior to film deposition, the silica wafers were cleaned with Acetone, Methonal, Isopropenal and de-ionized (DI) water for 5 minutes each in sequence, followed by drying with compressed nitrogen gas. A $25 \mathrm{~nm}$ adhesion Ti layer and $50 \mathrm{~nm}$ current-collected $\mathrm{Cu}$ layer were deposited on one side of the wafers via physical vapor deposition (PVD) at working pressure below $2 \times 10^{-6}$ Torr. Then, a Sn anode layer with thickness between $200 \mathrm{~nm}$ and $2 \mu \mathrm{m}$ was electroplated on the $\mathrm{Cu}$ layers with a commercial Sn electroplating solution (Solderon SC, produced by Rohm \& Haas). Before electroplating, the wafers were etched by $98 \%$ sulfuric acid to remove the native oxide layer on the surface. After the Sn film growth, the samples were cleaned with acetone and DI water in sequence for 5 minutes, and then dried with compressed nitrogen gas. Post sample fabrication, the samples were stored in an Ar-filled glove box, and both moisture and oxygen were below $0.1 \mathrm{ppm}$. A 0.5 mm thick Li foil was used as both counter and reference electrode. The separator was Celgard C480 (Celgard Inc., Charlotte, NC). The electrolyte composition was 1.2 M LiPF6 in EC/DEC with ratio 1:2 (wt. \%) (BASF Corp. A6 Series). The electrochemical measurements were done using Multistat 1470E (Solartron Analytical) at room temperature.

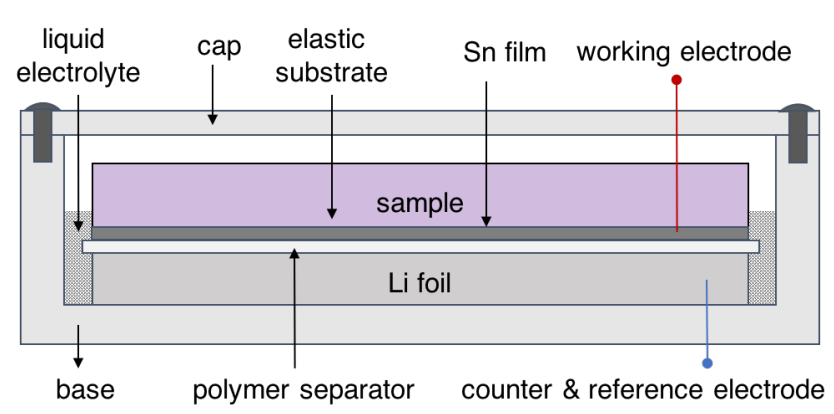

Figure 1: A schematic representation of the Li-Sn thin film system used in our experiments. The apparatus consists of a Li-ion half-cell with a thin-film Sn working electrode on silica quartz wafer substrate and a Li metal foil counter-electrode.

Prior to all experiments, the $\mathrm{Sn}$ electrode was first lithiated galvanostatically with $\mathrm{C} / 20$ rate from open-circuit potential at $2.75 \mathrm{~V}$ to $0.8 \mathrm{~V}$ and then the potential was held at $0.8 \mathrm{~V}$ 
for 20 hours to form a stable SEI layer to minimize the influence of the side reactions. We then conducted three kinds of experiments, PITT [30], Potentiostatic lithiation/delithiation, and Galvanostatic lithiation/delithiation.

PITT was done to study the Sn phase. The potential was decreased from $0.8 \mathrm{~V}$ (after SEI growth phase) in steps of $0.02 \mathrm{~V}$ till $0.7 \mathrm{~V}$ at which the $\mathrm{Li}_{2} \mathrm{Sn}_{5}$ phase nucleated (Figure 2a). In each step, the potential applied was held until the current fell below $0.05 \mathrm{~mA} \mathrm{~g}^{-1}$ (about $\mathrm{C} / 1800$ ). The transient current evolution following the voltage steps is used to determine the diffusivity and the Butler-Volmer parameter as discussed in detail in Section 6. In addition, the free-energy of Li-Sn as a function of Li concentration was determined from the steady-state voltage-charge measurements.

To determine the exchange current density of $\mathrm{Li}_{2} \mathrm{Sn}_{5}$, the potential was lowered to $0.65 \mathrm{~V}$ to nucleate the $\mathrm{Li}_{2} \mathrm{Sn}_{5}$ phase and grow it to approximately half the film thickness. The film was then allowed to reach equilibrium. Subsequently, a step voltage change was applied (from the equilibrium voltage) and the transient current was used to determine the Butler-Volmer parameter.

Galvanostatic lithiation/delithiation was used to determine the interface mobility. First, the potential was stepped down from $0.8 \mathrm{~V}$ to $0.665 \mathrm{~V}$, sufficiently low to nucleate and grow the $\mathrm{Li}_{2} \mathrm{Sn}_{5}$ phase. Then, the film was lithiated/delithiated at currents from $\mathrm{C} / 2500$ to $\mathrm{C} / 625$ allowing to it equilibriate in between. The plateau potential as a function of the current was used to determine the interface mobility.

Figure 2 shows representative current and voltage evolution with time in PITT and Potentiostatic experiments and Table 1 lists details of the various experiments used in the calibration.
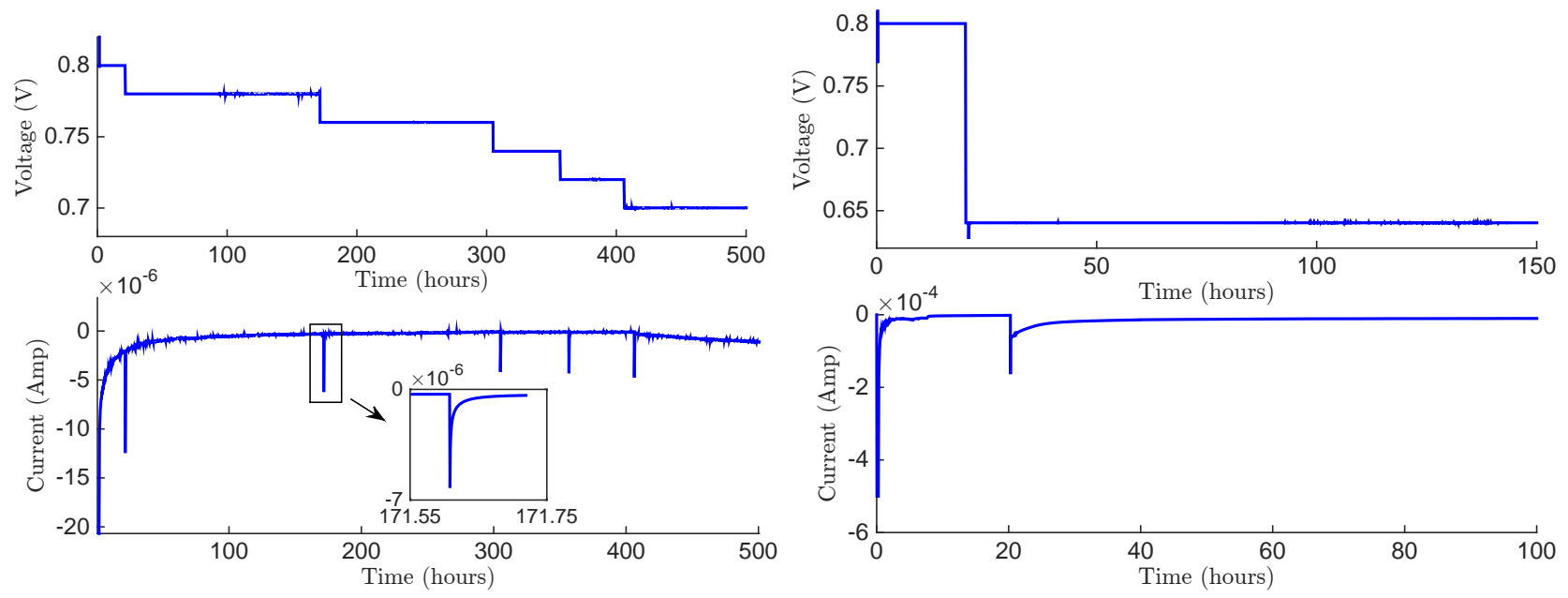

(a)

(b)

Figure 2: Voltage and current evolution in (a) PITT and (b) Potentiostatic experiments. These and other similar experiments (see Table 1 and Section 6) are used to determine the parameters in the Cahn-Hilliard model and compare its predictions.

\begin{tabular}{|c|c|c|c|}
\hline Experiment & Parmeters calibrated & Film thickness & Range \\
\hline PITT & $\begin{array}{c}D, M_{0}, i_{0} \text { of } \mathrm{Sn} \\
\text { Free-energy } G_{0}(c)\end{array}$ & $187 \mathrm{~nm}$ & $0.8 \mathrm{~V}$ to $0.7 \mathrm{~V}$ - steps of $0.02 \mathrm{~V}$ \\
\hline Potentiostatic & $i_{0}$ of $\mathrm{Li}_{2} \mathrm{Sn}_{5}$ & $1.85 \mu \mathrm{m}$ & $0.7 \mathrm{~V}, 0.8 \mathrm{~V}$ \\
\hline Galvanostatic & $i_{0}, \chi$ & $1.85 \mu \mathrm{m}$ & $\mathrm{C} / 2500$ to $\mathrm{C} / 625$ \\
\hline
\end{tabular}

Table 1: Experiments used to determine the parameters in the Cahn-Hilliard model. See Sections 3 and 6 for more details. 


\section{Phase field model of Li-Sn thin film electrodes}

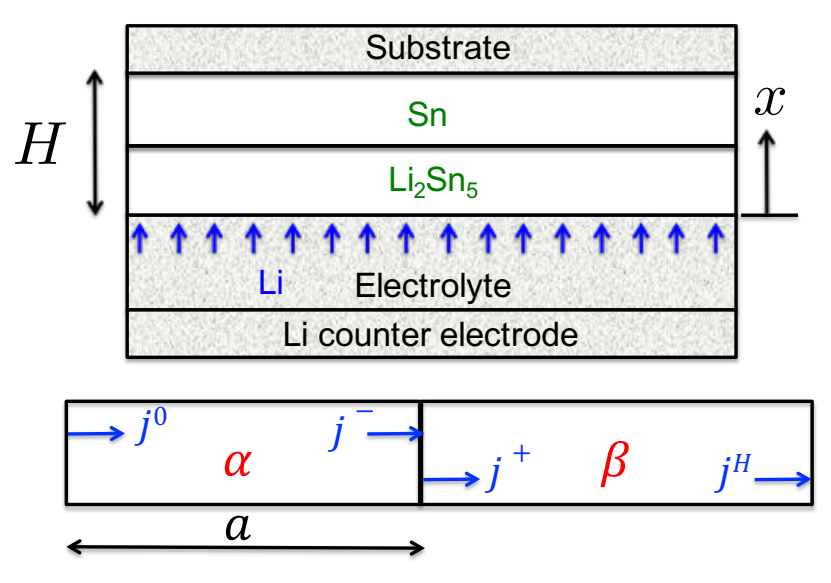

(a)

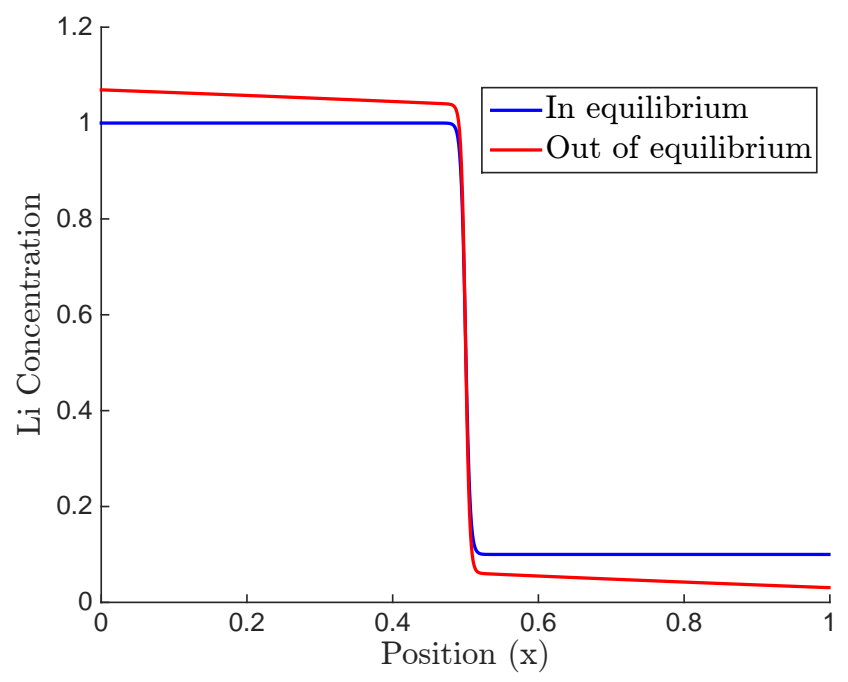

(b)

Figure 3: (a) Schematic representing the one-dimensional modeling of the thin-film experiments. (b) Li concentration profiles in the film at equilibrium (blue) and out of equilibrium due to a flux of Li (red). In Cahn-Hilliard models, concentration is also the phase variable.

We are interested in modeling the behavior of half-cells with a Li-Sn thin film electrode as illustrated in Figure 3a. We assume infinitely fast transport of Li ions in the electrolyte. The electrode initially consists of the pure Sn phase. Upon the application of an external electrical connection between the electrodes, $\mathrm{Li}$ is inserted into the $\mathrm{Sn}$ electrode through an electrochemical reaction at the electrode/electrolyte interface. We assume that Sn atoms are immobile and $\mathrm{Li}$ atoms diffuse through the film and occupy interstitial positions. When the $\mathrm{Li}$ concentration at the electrode surface reaches a critical value, the second phase nucleates and a phase boundary begins propagating through the film. Stresses accompanying lithiation and phase transformations in Li-Sn are of the order of a few $\mathrm{MPa}$ and we assume they do not significantly affect Li diffusion or interface propagation [31].

The Li transport, phase nucleation, and interface motion are modeled using a one-dimensional (along the thickness of the film) version of the Cahn-Hilliard equation.

$$
\frac{\partial c}{\partial t}=\frac{\partial}{\partial x} M \frac{\partial \mu}{\partial x}, \quad \mu=\frac{d G_{0}}{d c}-\kappa \frac{\partial^{2} c}{\partial x^{2}}
$$

where $c=\rho_{\mathrm{Li}} / \rho_{\mathrm{Sn}}$ (ratio of molar densities of $\mathrm{Li}$ and $\mathrm{Sn}$ ), $\mu$ is the chemical potential of $\mathrm{Li}, M$ is a function that characterizes the mobility of $\mathrm{Li}, \kappa$ is a parameter related to the interface-energy (determines the phase-boundary width), and $G_{0}(c)$ is the homogeneous free-energy of Li-Sn.

We consider a homogeneous free-energy of the form

$$
G_{0}(c)=\mu^{\mathrm{eq}} c+\frac{W}{2\left(\Delta c_{0}\right)^{2}}\left(c-c_{0}^{\alpha}\right)^{2}\left(c-c_{0}^{\beta}\right)^{2}
$$

where $c_{0}^{\alpha}$ and $c_{0}^{\beta}$ are the equilibrium concentrations of the two phases, $\Delta c_{0}=c_{0}^{\beta}-c_{0}^{\alpha}$, $\mu^{\text {eq }}$ is the equilibrium chemical potential, and $W$ is free-energy curvature at the equilibrium concentrations. The quartic double-well energy has been used in many phase-field studies. The linear term $\mu^{\mathrm{eq}} c$ is necessary to get the right plateau voltage between the two phases (see Section 6). An advantage of this free-energy is that it is amenable to perturbation analysis (Section 4.2). Figure 4 shows the free-energy $G_{0}(c)$ and $d G_{0} / d c$ for $c_{0}^{\alpha}=0.1, c_{0}^{\beta}=1, \mu^{\mathrm{eq}}=1, W=50$. The 
free-energy determines the equilibrium chemical potential, spinodal and metastable regions, and the nucleation potentials for the phase transformations (see [30] for more).

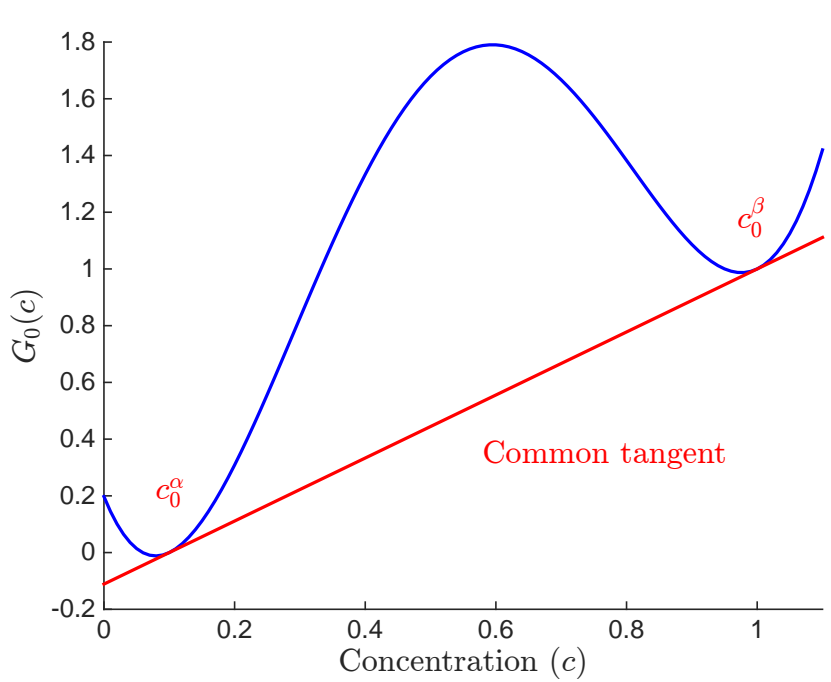

(a)

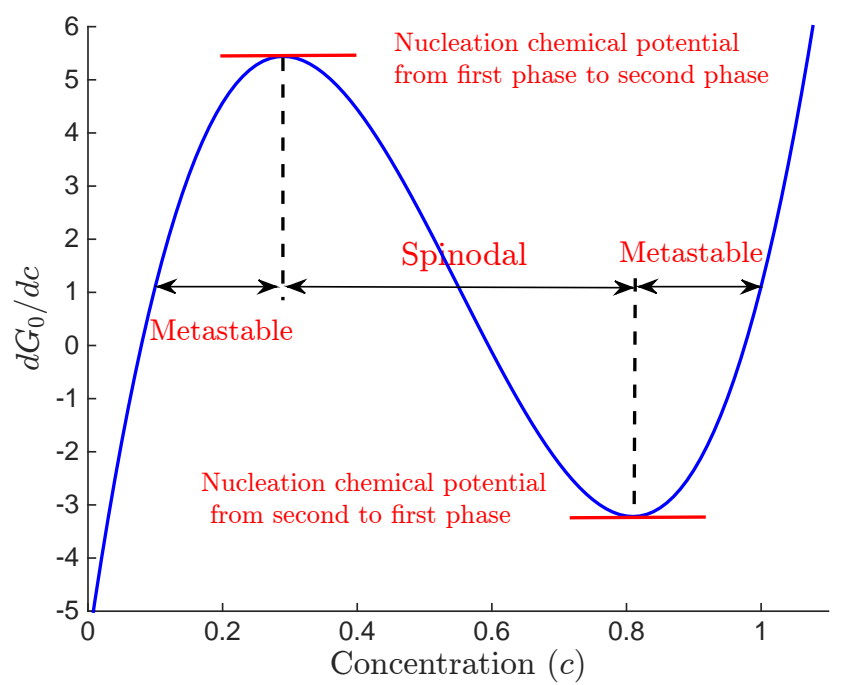

(b)

Figure 4: (a) Homogeneous free-energy $G_{0}$ (Equation 2) and (b) its derivative $d G_{0} / d c$. Each energy minimum corresponds to a stoichiometric phase. The common tangent for equilibrium, the spinodal and metastable regions, and the nucleation potentials for phase transformations are all shown.

We assume the mobility $M$ in Equation 1 to depend on the concentration gradient (see Sections 4 and 5 for more details)

$$
M=\frac{M_{0}}{1+\frac{\chi}{\Delta c_{0}}\left|\frac{\partial c}{\partial x}\right|} .
$$

Here, $M_{0}$ is the Li mobility in the bulk phase and $\chi$ is a parameter that determines the interface mobility. In the bulk, concentration gradients are small, $M \approx M_{0}$ and the chemical diffusivity of Li is given by $D=M_{0}\left(d^{2} G_{0} / d c^{2}\right)$ (this can be seen by linearizing the Cahn-Hilliard equation). $\chi=0$ results in infinite interface mobility while for a nonzero $\chi$, the interface mobility is finite (see Section 5 for details).

To predict the electrochemical response, we need to model the Li insertion reaction, and possibly side-reactions. As a simple preliminary approximation, we ignore side reactions and suppose that the electrode current is related to the over-potential by the Butler-Volmer equation $[32]$

$$
i=i_{0}\left(e^{\frac{\alpha \eta F}{R T}}-e^{-\frac{(1-\alpha) \eta F}{R T}}\right)
$$

where $i$ is the current density, $i_{0}$ is the exchange current density, which may be a function of the Li concentration at the surface of the electrode (as well as the Li ion concentration in the electrolyte), $\alpha$ is a phenomenological constant (between 0 and 1, we assume the anodic and cathodic transfer coefficients to be $\alpha$ and $1-\alpha$ respectively), $F$ is the Faraday constant, $R$ is the universal gas constant, and $T$ is temperature. $\eta=V-U_{0}$ is the overpotential (the difference between the externally applied potential $\mathrm{V}$ and the rest potential $U_{0}$ ). The rest potential is related to the chemical potential at the surface of the electrode by

$$
U_{0}=-\frac{1}{F}\left(\mu^{\theta}+\mu\right)
$$

where $\mu^{\theta}$ is a (constant) reference potential (its value depends on the choice of counter-electrode used to define the electric potential). The electrode current $I$ is related to flux $J$ at the electrode/electrolyte interface by $I=i A=-A \rho_{\mathrm{Sn}} F J$, where $A$ is the area of the film. 
The remaining boundary conditions are: $\frac{\partial \mu}{\partial x}=0$ at $x=H$ since the flux at the substrate is zero. At the electrode/electrolyte interface $x=0,-M \frac{\partial \mu}{\partial x}=J$. We also assume $\frac{\partial c}{\partial x}=0$ at $x=0, H$.

\section{Sharp-interface model}

We find it useful to study the sharp-interface limit of the governing equations 1-3. This reveals the interface behavior and is helpful in finding model parameters from experiments. We start by deriving a general energetic formulation of a sharp-interface model that identifies the kinetic variables and their conjugate forces for diffusion in the bulk and at the interface. Then we present the results of a perturbration analysis of the governing quations 1-3. Comparing the two identifies the kinetic relations for the interface implied by the Cahn-Hilliard equations.

\subsection{A general sharp-interface model}

Consider a thin film of thickness $H$ in which two phases $\alpha$ and $\beta$ are separated by an interface at $x=a$ (Figure 3a). The total energy of the system is given by

$$
U=A\left[\int_{0}^{a} G_{\alpha}(c(x)) \mathrm{d} x+\int_{a}^{H} G_{\beta}(c(x)) \mathrm{d} x\right]
$$

where $G_{\alpha}$ and $G_{\beta}$ are the free-energy densities of the two phases, $A$ is the cross-sectional area, $a$ is the position of the interface, and $H$ is the thickness of the film. We have ignored the surface energy since it does not change with time. Differentiating Equation 6 with respect to time,

$$
\dot{U}=A\left[\int_{0}^{a} \frac{d G_{\alpha}}{d c} \frac{\partial c}{\partial t} \mathrm{~d} x+\int_{a}^{H} \frac{d G_{\beta}}{d c} \frac{\partial c}{\partial t} \mathrm{~d} x+\left\{G_{\alpha}\left(c^{-}\right)-G_{\beta}\left(c^{+}\right)\right\} \frac{d a}{d t}\right]
$$

Using $\mu=d G / d c, \partial c / \partial t=-\partial j / \partial x$ (where $j$ is the flux) and integrating by parts, we can write this as

$$
\dot{U}=A\left[\mu^{0} j^{0}-\mu^{H} j^{H}+\int_{0}^{H} j \frac{\partial \mu}{\partial x} \mathrm{~d} x+\mu^{+} j^{+}-\mu^{-} j^{-}+\left\{G_{\alpha}\left(c^{-}\right)-G_{\beta}\left(c^{+}\right)\right\} \frac{d a}{d t}\right]
$$

where $\mu^{0}, \mu^{H}, j^{0}, j^{H}$ are the chemical potentials and fluxes at $x=0$ and $x=H$ respectively. The first two terms capture energy flux at the boundaries, the integral is the dissipation in the bulk due to diffusion, and the rest correspond to dissipation at the interface. The superscripts $+/-$ label states just ahead and behind the interface. The interfacial dissipation is

$$
\dot{U}_{\text {int }}=A\left[\mu^{+} j^{+}-\mu^{-} j^{-}+\left\{G_{\alpha}\left(c^{-}\right)-G_{\beta}\left(c^{+}\right)\right\} \frac{d a}{d t}\right]
$$

Using

$$
\mu^{+} j^{+}-\mu^{-} j^{-}=\frac{\mu^{+}+\mu^{-}}{2}\left(j^{+}-j^{-}\right)+\left(\mu^{+}-\mu^{-}\right) \frac{j^{+}+j^{-}}{2}
$$

and the conservation equation

$$
\frac{d a}{d t}\left(c^{+}-c^{-}\right)=j^{+}-j^{-},
$$

the interfacial dissipation can be written as

$$
\dot{U}_{\mathrm{int}}=A\left[\frac{\mu^{+}-\mu^{-}}{2}\left(j^{+}+j^{-}\right)+\left\{\frac{\left(\mu^{+}+\mu^{-}\right)}{2}-\frac{G_{\beta}\left(c^{+}\right)-G_{\alpha}\left(c^{-}\right)}{c^{+}-c^{-}}\right\}\left(j^{+}-j^{-}\right)\right]
$$


This identifies the driving forces conjugate to the average flux and interface velocity (the jump in flux is proportional to the interface velocity). The interface kinetics is defined by prescribing the velocity and average flux as a function of their driving forces. Assuming linear kinetics,

$$
j^{+}+j^{-}=-K_{1}\left(\mu^{+}-\mu^{-}\right), \quad j^{+}-j^{-}=-2 K_{2}\left\{\frac{\left(\mu^{+}+\mu^{-}\right)}{2}-\frac{G_{\beta}\left(c^{+}\right)-G_{\alpha}\left(c^{-}\right)}{c^{+}-c^{-}}\right\} .
$$

The conditions $K_{1}>0, K_{2}>0$ ensure that the interfacial dissipation is always positive. At equilibrium, setting the driving forces to zero, we have

$$
\mu^{e q+}=\mu^{e q-}=\frac{d G_{\alpha}}{d c}\left(c^{e q-}\right)=\frac{d G_{\beta}}{d c}\left(c^{e q+}\right)=\frac{G_{\beta}\left(c^{e q+}\right)-G_{\alpha}\left(c^{e q-}\right)}{c^{e q+}-c^{e q-}} .
$$

At equilibrium, the chemical potential is continuous across the interface and is given by the common tangent rule. For small deviations about equilibrium, linearizing the kinetic relations (Equation 13),

$$
j^{+}+j^{-}=-K_{1}\left(\delta \mu^{+}-\delta \mu^{-}\right), \quad j^{+}-j^{-}=-K_{2}\left(\delta \mu^{+}+\delta \mu^{-}\right)
$$

where $\delta \mu^{+}, \delta \mu^{-}$are the deviations of the chemical potential from their equilibrium value. The average flux at the interface is driven by the jump in the chemical potential while the jump in the flux, which is proportional to the interface velocity, is driven by the sum of the deviations of the chemical potential from equilibrium. The interfacial dissipation for small deviations about equilibrium is

$$
\dot{U}_{\mathrm{int}}=-A\left[K_{1} \frac{\left(\delta \mu^{+}-\delta \mu^{-}\right)^{2}}{2}+K_{2} \frac{\left(\delta \mu^{+}+\delta \mu^{-}\right)^{2}}{2}\right]=-A\left[\frac{\left(j^{+}+j^{-}\right)^{2}}{2 K_{1}}+\frac{\left(j^{+}-j^{-}\right)^{2}}{2 K_{2}}\right]
$$

If flux in the bulk is proportional to the chemical potential gradient,

$$
j=-M \frac{\partial \mu}{\partial x}, \quad M>0,
$$

bulk dissipation is always positive. The bulk dissipation is

$$
\dot{U}_{\text {bulk }}=A \int_{0}^{H} j \frac{\partial \mu}{\partial x} \mathrm{~d} x=-A \int_{0}^{H} \frac{j^{2}}{M} \mathrm{~d} x .
$$

\subsection{Perturbation analysis of Cahn-Hilliard equations}

With the above general formulation, let us turn our attention back to the Cahn-Hilliard equations. Here, we borrow the results from the perturbation analysis of Langer and Sekerka [32] and refer the reader to that paper for details. Langer and Sekerka studied the nonequilibrium interface behavior for small perturbations about its equilibrium (of equations 1-3).

When the interface is at equilibrium, the concentrations at its boundaries reach the equilibrium values $c_{0}^{\alpha}, c_{0}^{\beta}$ given by the common tangent rule (Figure 4 ). When it is pushed out of equilibrium, it is moving and/or there is a flux through it, the concentrations deviate from $c_{0}^{\alpha}, c_{0}^{\beta}$ (Figure $3 \mathrm{~b}$ ). Langer and Sekerka showed that the deviations as a function of the interface velocity and flux are

$$
\delta c^{-}=-\frac{\left[c_{0}\right] \chi}{12 M_{0} W} v+\frac{\chi}{4 M_{0} W}\left(j^{-}+j^{+}\right), \quad \delta c^{+}=-\frac{\left[c_{0}\right] \chi}{12 M_{0} W} v-\frac{\chi}{4 M_{0} W}\left(j^{-}+j^{+}\right)
$$

where $\delta c^{-}, \delta c^{+}$are the concentration deviations from equilibrium, $j^{-}, j^{+}$are the fluxes at the interface boundaries, $v$ is the interface velocity, and $\left[c_{0}\right]$ is the jump in the equilibrium 
concentration. Using the jump conservation equation $v[c]=[j]$ and approximating $[c]$ by $\left[c_{0}\right]$ for small deviations from equilibrium, the above equations can be written as

$$
\delta c^{-}=\frac{\chi}{3 M_{0} W} j^{-}+\frac{\chi}{6 M_{0} W} j^{+}, \quad \delta c^{+}=-\frac{\chi}{6 M_{0} W} j^{-}-\frac{\chi}{3 M_{0} W} j^{+}
$$

For small deviations about the equilibrium, $\delta \mu=W \delta c$. Using this,

$$
\delta \mu^{-}=\frac{\chi}{3 M_{0}} j^{-}+\frac{\chi}{6 M_{0}} j^{+}, \quad \delta \mu^{+}=-\frac{\chi}{6 M_{0}} j^{-}-\frac{\chi}{3 M_{0}} j^{+}
$$

In the sharp-interface limit, one can think of these equations as boundary conditions at the interface. To determine the evolution of the system, the diffusion equations in the bulk have to be solved coupled with the above equations at the interface.

These results allow us to interpret roles of $\chi$ and $M_{0}$ in Equation 3. If $\chi=0, M=M_{0}$ and Equation 1 reduces to the standard Cahn-Hilliard equation; $\delta c^{+/-}=0, \delta \mu^{+/-}=0$ and in the sharp-interface limit, the interface is always in local equilibrium. For a nonzero $\chi$, $\delta c^{+/-} \neq 0, \delta \mu^{+/-} \neq 0$ and depend on the velocity and the flux. The number $\chi$ thus characterizes the interface-mobility.

\subsection{Kinetic relations in the Cahn-Hilliard model}

From the general model and the perturbation analysis, we can infer the kinetic relations implied by the sharp-interface limit of the Cahn-Hilliard equations. We can write the results of the perturbation analysis (Equations 21) as:

$$
j^{+}+j^{-}=-\frac{2 M_{0}}{\chi}\left(\delta \mu^{+}-\delta \mu^{-}\right), \quad j^{+}-j^{-}=-\frac{6 M_{0}}{\chi}\left(\delta \mu^{+}+\delta \mu^{-}\right) .
$$

Comparing these with the kinetic relations (Equations 15),

$$
K_{1}=\frac{2 M_{0}}{\chi}, \quad K_{2}=\frac{6 M_{0}}{\chi} .
$$

This shows that the Cahn-Hilliard equations are a particular case of a more general class of kinetic laws where $K_{2}=3 K_{1}$. Further, for constant kinetic parameter $(\chi \rightarrow 0), K_{1}, K_{2} \rightarrow \infty$ and the interfacial dissipation goes to zero for any finite flux (Equation 16). Thus the standard Cahn-Hilliard formulation implies zero energy dissipation due to interface propagation.

\subsection{Steady diffusion}

Let us consider the case when the lithiation rate is small enough that bulk diffusion is at steady state. The chemical potentials in the bulk on either side of the phase boundary are linear. At the interface, the chemical potentials are given by the perturbation analysis (Equation 21). If $\mu^{0}$ and $\mu^{H}$ are the chemical potentials at $x=0$ and $x=H$, then the fluxes in the bulk are

$$
j^{-}=-M_{0} \frac{\mu^{-}-\mu^{0}}{x_{\mathrm{int}}}, \quad j^{+}=-M_{0} \frac{\mu^{H}-\mu^{+}}{H-x_{\mathrm{int}}} .
$$

Flux at the substrate is zero, $j^{+}=0$. Using the perturbation analysis solutions (Equation 21), $\mu^{-}=\mu^{\mathrm{eq}}+\delta \mu^{-}$, and the above equations,

$$
\mu^{0}-\mu^{\mathrm{eq}}=\frac{x_{\mathrm{int}}+\chi / 3}{M_{0}} j^{-}
$$


This allows us to determine material parameters $M_{0}$ and $\chi$ from electrochemical experiments. Linearizing the Butler-Volmer equation (see equations 4 and 5) for small currents,

$$
-\rho_{\mathrm{Sn}} F J=i=i_{0} \frac{\eta F}{R T}=i_{0} \frac{V F+\mu^{\theta}+\mu^{0}}{R T}
$$

where $J$ the flux at the surface. Using the above two equations,

$$
V=-\frac{\mu^{\theta}+\mu^{\mathrm{eq}}}{F}+\left[\frac{x_{\mathrm{int}}}{F M_{0} \rho_{\mathrm{Sn}}}+\frac{\chi}{3 F M_{0} \rho_{\mathrm{Sn}}}+\frac{R T}{i_{0}}\right] i
$$

In a galvanostatic experiment, $i$ is a constant. The voltage $V$ varies linearly with the interface position $x_{\text {int }}$ (for given $i$ ) and the current $i$ (for given $x_{\text {int }}$ ). These can be used to determine the kinetic parameter $M_{0}$ and the interface-mobility parameter $\chi$ if we know the Butler-Volmer constant $i_{0}$ (see Section 6).

\section{Model behavior}

Let us now look at a few representative examples that elucidate the general behavior of the modified Cahn-Hilliard model. Using the film thickness $H$, a typical relaxation time $t^{*}=10$ hours (see Figure 2), and $R T$ to nondimensionalize length, time, and energy respectively, the governing equations take the form

$$
\begin{gathered}
\bar{\mu}=\frac{d \bar{G}_{0}}{d c}-\bar{\kappa} \frac{\partial^{2} c}{\partial \bar{x}^{2}}, \quad \frac{\partial c}{\partial \bar{t}}=\frac{\partial}{\partial \bar{x}} \bar{M} \frac{\partial \bar{\mu}}{\partial \bar{x}}, \quad \bar{M}=\frac{\bar{M}_{0}}{1+\frac{\bar{\chi}}{\Delta c_{0}}\left|\frac{\partial c}{\partial \bar{x}}\right|} \\
\frac{\partial \bar{\mu}}{\partial \bar{x}}=0 \text { at } \bar{x}=1, \quad-\bar{M} \frac{\partial \bar{\mu}}{\partial \bar{x}}=\bar{J} \text { at } \bar{x}=0, \quad \frac{\partial c}{\partial \bar{x}}=0 \text { at } \bar{x}=0,1 \\
\bar{J}=\bar{i}=\bar{i}_{0} e^{\alpha \bar{\eta}}\left(1-e^{-\bar{\eta}}\right), \quad \bar{\eta}=\bar{V}-\bar{U}_{0}, \quad \bar{U}_{0}=-\left(\bar{\mu}^{\theta}+\bar{\mu}\right)
\end{gathered}
$$

where

$$
\begin{gathered}
{\left[\bar{G}_{0}, \bar{\mu}, \bar{\mu}^{\theta}\right]=\left[G_{0}, \mu, \mu^{\theta}\right] \frac{1}{R T}, \quad \bar{\kappa}=\frac{\kappa}{R T H^{2}}, \quad \bar{M}=M \frac{R T t^{*}}{H^{2}},} \\
\bar{J}=J \frac{t^{*}}{H}, \quad \bar{I}=I \frac{t^{*}}{F \rho_{\mathrm{Sn}} A H}, \quad\left[\bar{i}, \bar{i}_{0}\right]=\left[i, i_{0}\right] \frac{t^{*}}{F \rho_{\mathrm{Sn}} H}, \quad\left[\bar{\eta}, \bar{V}, \bar{U}_{0}\right]=\left[\eta, V, U_{0}\right] \frac{F}{R T} .
\end{gathered}
$$

Equation 27 after nondimensionalization is

$$
\bar{V}=-\bar{\mu}^{\theta}-\bar{\mu}^{\mathrm{eq}}+\left[\frac{\bar{x}_{\mathrm{int}}}{\bar{M}_{0}}+\frac{\bar{\chi}}{3 \bar{M}_{0}}+\frac{1}{\bar{i}_{0}}\right] \bar{I}
$$

\subsection{Galvanostatic lithiation}

Let us first look at galvanostatic lithiation (see Figure 3 a for a schematic of the system). Initially, the Li concentration in the film is uniform $(c=0)$. We start inserting $\mathrm{Li}$ at $\bar{x}=0$ at a constant flux $\bar{J}$. The concentration gradually increases and when it reaches the (lower) spinodal concentration at the boundary $\bar{x}=0$, the new phase nucleates. Upon further lithiation, the phase boundary propagates into the film. The concentration profiles just before nucleation and during phase propagation are shown in Figure 5. During delithiation, the low concentration phase nucleates at the boundary $\bar{x}=0$. If the applied current is small enough that the concentration at $\bar{x}=0$ does not reach the (higher) spinodal concentration, the existing phase boundary is pulled toward $\bar{x}=0$. 


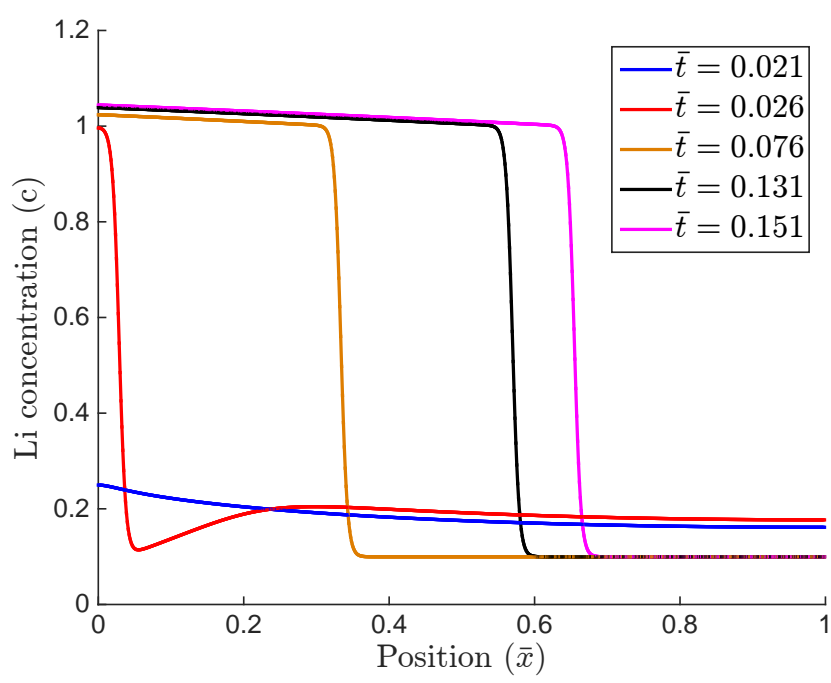

(a)

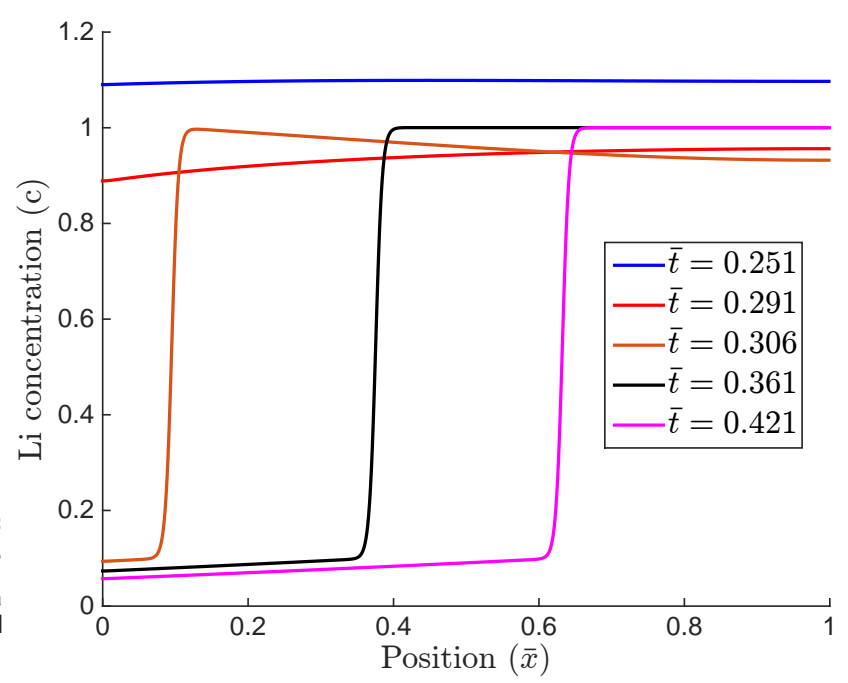

(b)

Figure 5: Snapshots of concentration profile during galvanostatic (a) lithiation and (b) delithiation. In both cases, a new phase nucleates at $\bar{x}=0$ and propagates into the film. The nucleation occurs when the concentration at $\bar{x}=0$ reaches that at the boundary of the spinodal region (see Figure 4).

The voltage evolution during galvanostatic lithiation is shown in Figure 6a for three different values of the applied flux $\bar{J}$. Initially, as we start inserting $\mathrm{Li}$, the voltage decreases until nucleation of the new phase. During nucleation, the voltage increases as the concentration at the boundary increases to that of the new phase resulting in a characteristic bump. The slope of the voltage-capacity during subsequent phase propagation increases with increasing flux. This is because the slope of the chemical potential in the bulk is (at steady-state) proportional to the flux $\bar{J}$, larger currents lead to larger chemical potential change requiring lower voltages to keep inserting $\mathrm{Li}$ at the same rate. The deviation of voltage from the equilibrium voltage (here $\bar{V}^{\text {eq }}=-1$ ) during phase propagation also depends on the Butler-Volmer parameter $\bar{i}_{0}$, here we have used $\bar{i}_{0}=1$.

The above described features of a voltage bump during nucleation and increasing slope of voltage-capacity with increasing flux is observed in the experiments as well. For example, Figure 6b shows experimentally measured voltage evolution during galvanostatic lithiation/delithiation for Li-Si/Li-Sn mixed-matrix electrode [33].

\subsection{Potentiostatic lithiation}

Potentiostatic lithiation is very useful in determining many material properties such as the freeenergy, diffusivity, and interface-mobility. A representative potentiostatic lithiation simulation is shown in Figure 7. We start with a thin film in the first phase, the Li concentration is $c=0$. We then instantaneously lower the voltage to $\bar{V}=\bar{V}_{1}$.

After the voltage step, as we start inserting Li, the concentration and chemical potential increase in the film and the current starts to drop. If the concentration at the boundary reaches the (lower) spinodal concentration (Figure 4), a new phase nucleates and propagates into the film. Three snapshots of chemical potential in the film during phase propagation are shown in Figure 7a. All the chemical potential drop across the film happens only within the first phase. In particular, the chemical potential is continuous across the phase-boundary and its value there is equal to the equilibrium chemical potential (which is 1 here). This can also be seen from the perturbation analysis (Equation 21) where $\bar{\chi}=0 \Longrightarrow \delta \bar{\mu}=0$. This demonstrates that in the standard Cahn-Hilliard model, the interface has infinite mobility and is always in 


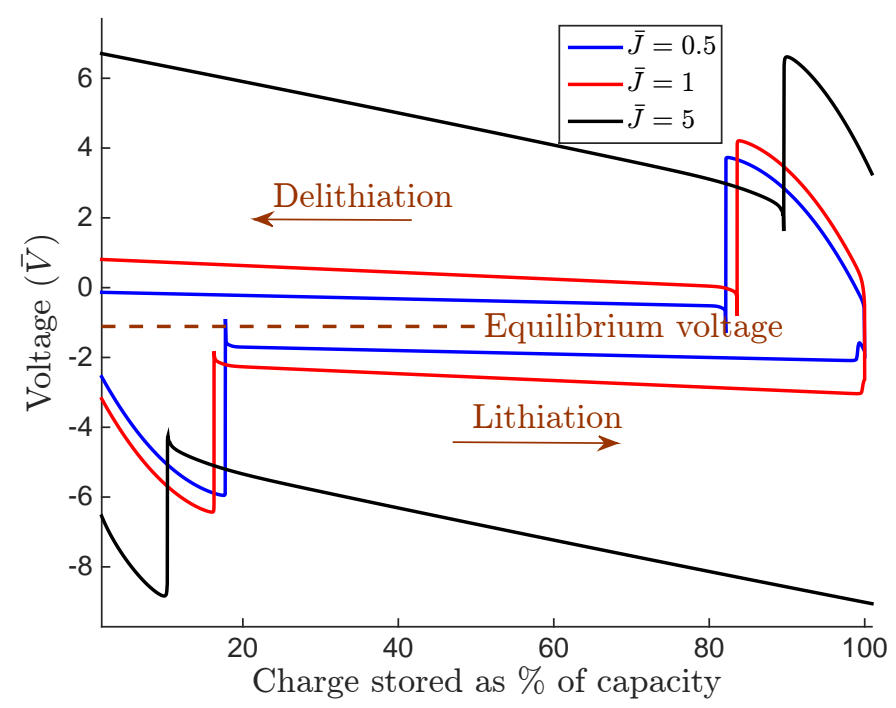

(a)

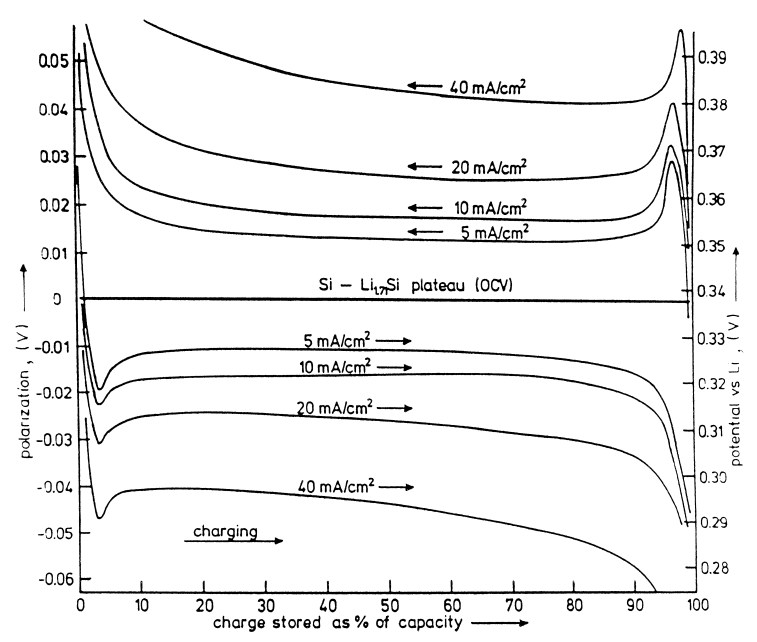

(b)

Figure 6: Voltage evolution during galvanostatic lithiation/delithiation in (a) our simulations (b) experiments on a Li-Si/Li-Sn mixed-matrix electrode [33] (reproduced by permission of The Electrochemical Society). The characteristic bump in the voltage corresponds to nucleation of a phase. The slope during phase propagation increases with increasing current due to diffusionlimited lithiation.

local equilibrium.

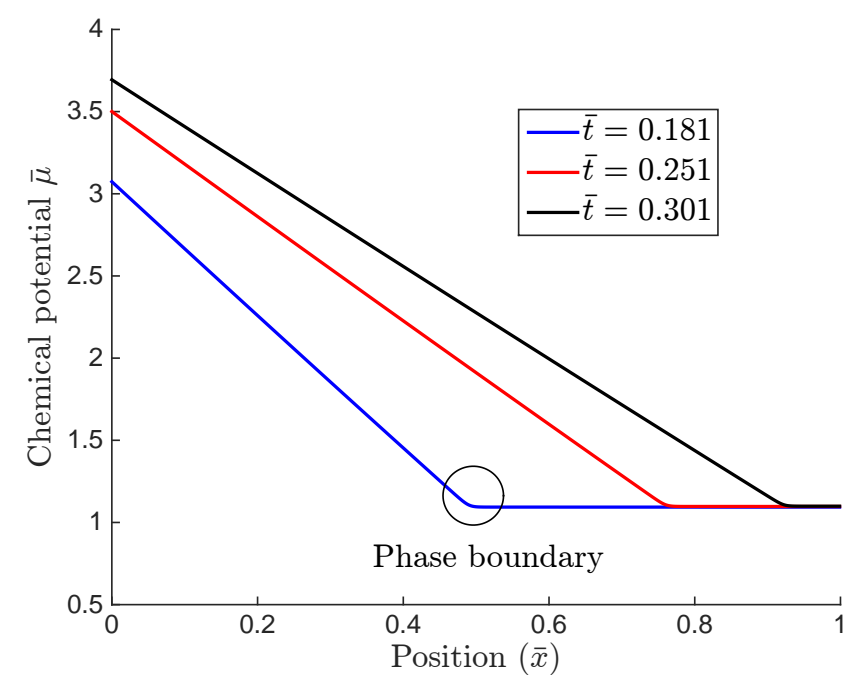

(a)

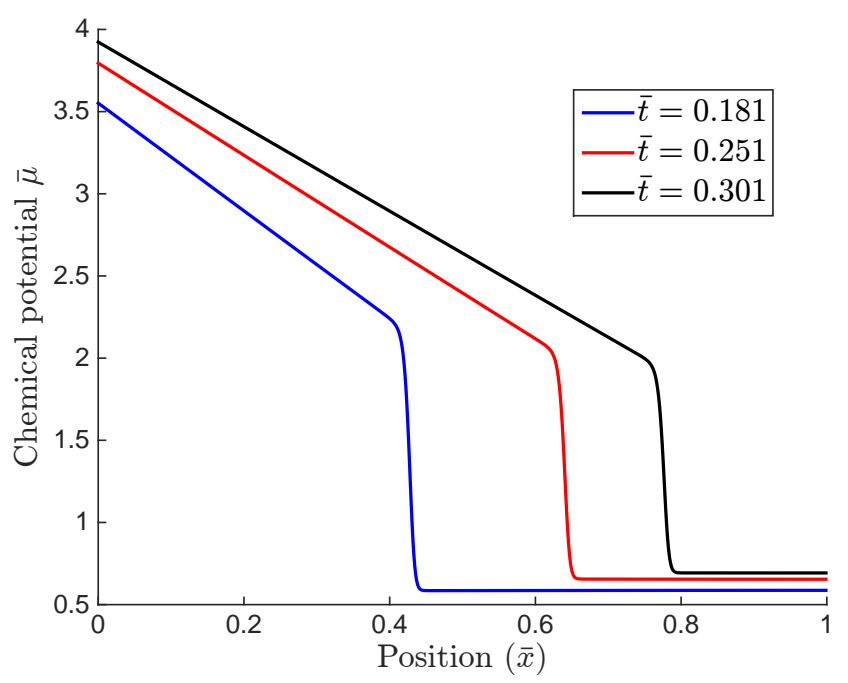

(b)

Figure 7: Three snapshots of chemical potential during phase propagation. (a) $\bar{\chi}=0$, all the chemical potential drop across the film happens across the first phase. The jump in potential across the interface is zero and the potential there is equal to the equilibrium potential. This demonstrates that the interface in standard Cahn-Hilliard equations is always in local equilibrium. (b) $\bar{\chi}=0$, the chemical potential is now discontinuous across the phase boundary. The interface is out of equilibrium, the deviation of chemical potential from its equilibrium value acting as the driving force conjugate to interface velocity. With increasing $\bar{\chi}$, the interface becomes more sluggish, its velocity decreases, and it is pushed more out of equilibrium.

A concentration-gradient dependent Li mobility (nonzero $\bar{\chi}$ in Equation 3 ) gives the interface a finite mobility. We repeat the above potentiostatic lithiation simulation but change $\bar{\chi}$ 
from 0 to 1 . Figure $7 \mathrm{~b}$ shows again, three snapshots of chemical potential. Comparing with Figure $7 \mathrm{a}$, we observe two things. First, the chemical potential is now discontinuous across the interface. Behind the interface, $\mu>\mu^{\mathrm{eq}}$ while ahead of it $\mu<\mu^{\mathrm{eq}}$. As we saw earlier, the deviation of the chemical potential from its equilibrium value acts as the driving force conjugate to the interface velocity (Equation 22). The magnitude of the potential jump across the phase-boundary decreases during propagation since the current (flux) decreases (Equation 21). Second, as $\bar{\chi}$ increases, lithiation gradually becomes interface limited and the phase propagation velocity decreases. In the limiting case $\bar{\chi} \rightarrow \infty$, the interface is immobile and all the diffusing $\mathrm{Li}$ accumulates behind the phase-boundary. Thus, $\bar{\chi}=0$ and $\bar{\chi} \rightarrow \infty$ are limits of infinite mobility and immobility for the interface. This gives us the ability to model both diffusion-limited and interface-limited processes as opposed to the standard Cahn-Hilliard which can model only diffusion-limited lithiation.

There are three timescales: the surface-reaction timescale, the bulk-diffusion timescale, and the interface timescale. Apart from other factors like the film width $H$, the three are determined by $i_{0}, M_{0}, \chi$ respectively. We now turn our attention to finding these numbers for the Li-Sn system.

\section{Comparison of theory and experiment}

We have to determine the following parameters from experiments: the homogeneous freeenergy derivative $d G_{0} / d c$, the Butler-Volmer parameter $i_{0}$, the kinetic parameter $M_{0}$, and the interface-mobility parameter $\chi$. In calibrating these parameters, it is important to determine how much of the measured current in the experiment goes into the electrode and how much goes into SEI formation. To estimate this, we assume that all of the $\mathrm{Li}$ in the first 20 hours (during which the potential is held at $0.8 \mathrm{~V}$ ) goes into SEI and use an exponential fit for the last few hours of current evolution in this period. We assume that subsequent SEI growth follows this decay up to a constant residual value (equal to the residual current we observe in the later stages in our experiments),

$$
I(t)= \begin{cases}I_{0} e^{-\left(t-t_{\text {initial }}\right) / t_{\text {decay }},}, & \text { if } t \leq t_{\text {residual }} \\ I_{\text {residual }}, & \text { otherwise }\end{cases}
$$

where the values of $I_{0}, t_{\text {initial }}$ depend on the initial time from which the fit is made. $t_{\text {residual }}$ is the time at which $I_{0} e^{-\left(t-t_{\text {initial }}\right) / t_{\text {decay }}}$ reaches the residual current $I_{\text {residual }}$. Typical value of $t_{\text {decay }}$ in our experiments, the decay time for the SEI current, is about 30 hours. The SEI current at the end of 20 hours is of the order of $0.1 \mu \mathrm{A} \mathrm{cm}{ }^{-2}$. The residual current $I_{\text {residual }}$ observed is about $0.005 \mu \mathrm{A} \mathrm{cm}^{-2}$. This is significantly smaller than the currents used in our calibration. From the fit, we calculate the total charge loss to SEI (over the timescale of our experiments) to be about $0.05 \mathrm{C} \mathrm{cm}^{-2}$ which is the same as that reported by Bucci et al. [34] (although that is for $\mathrm{Si}$ ).

\subsection{Free-energy}

The homogeneous free-energy derivative $d G_{0} / d c$ is found from the steady-steady voltage vs charge in a PITT experiment (Figure 2a). After each voltage step, we allow the current to drop to a prescribed value $\left(0.05 \mathrm{~mA} \mathrm{~g}^{-1}\right.$, about $\left.\mathrm{C} / 1800\right)$. From the total charge, we calculate the equilibrium Li concentration at this voltage (these are preliminary results, an alternative method would be to let the system go to OCV and use the voltage corresponding to this state. In light of this, the numbers in Table 2 should be regarded only qualitatively. We have not used these numbers in calibrating our free-energy). Table 2 shows the results of such a 
calculation for the Sn phase. $c=0$ and $c=0.4$ correspond to pure Sn and the first phase $\mathrm{Li}_{2} \mathrm{Sn}_{5}$. The chemical potential can then be determined by setting the overpotential to zero in the Butler-Volmer equation.

$$
V=-\frac{1}{F}\left(\mu^{\theta}+\mu\right)=-U^{\theta}-\frac{1}{F} \frac{d G_{0}}{d c} \Longrightarrow \frac{d G_{0}}{d c}=-F\left(V+U^{\theta}\right),
$$

where $U^{\theta}=2.75 \mathrm{~V}$ is the open-circuit voltage with respect to $\mathrm{Li} / \mathrm{Li}^{+}$.

The free-energy parameters (Equation 2) are determined to satisfy the following conditions:

- Energy minima $\left(d G_{0} / d c=0\right)$ at $c=0$ and $c=0.4$ since these correspond to stoichiometric phases of $\mathrm{Sn}$ and $\mathrm{Li}_{2} \mathrm{Sn}_{5}$ (Figure $4 \mathrm{a}$ ). The curvatures at these concentrations must be positive since they are energy minima $\left(d^{2} G_{0} / d c^{2}>0\right)$.

- Plateau voltage between the first two phases is $0.75 \mathrm{~V}$.

- The (homogeneous) nucleation voltage for $\mathrm{Sn} \rightarrow \mathrm{Li}_{2} \mathrm{Sn}_{5}$ transformation is around $0.7 \mathrm{~V}$. With the first two constraints, the double-well free-energy predicts a nucleation potential significantly larger than that in experiment. Thus, we minimize the nucleation potential (this corresponds to the limiting condition of the $d^{2} G_{0} / d c^{2}=0$ at $c=0.4$ ).

The best-fit parameters based on the above conditions are:

$$
c_{0}^{\alpha}=0.0346, c_{0}^{\beta}=0.4853, \mu^{\mathrm{eq}}=0.193 \mathrm{MJ} \mathrm{mol}^{-1}, W=4.57 \mathrm{MJ} \mathrm{mol}^{-1} .
$$

The free-energy derivative based on the above numbers and that determined from experiment are shown in Figure 8. The double-well energy predicts a nucleation potential about twice as large as that observed in experiment. This suggests that free-energies such as Equation 2 , though convenient for analysis, may not be (quantitatively) good in capturing the concentration dependence of the free-energy. Predictive phase-field models require a better way of incorporating free-energy determined from experiments.

\begin{tabular}{|c|c|}
\hline Voltage $(\mathrm{V})$ & Concentration \\
\hline 2.7535 & 0 \\
\hline 0.78 & 0.1501 \\
\hline 0.76 & 0.1931 \\
\hline 0.74 & 0.2033 \\
\hline 0.72 & 0.2131 \\
\hline 2.7535 & 0.4 \\
\hline
\end{tabular}

Table 2: Voltage vs concentration at steady-state during PITT. The concentration is calculated from the total charge after correcting for loss to SEI.

\subsection{Diffusivity and exchange-current density of Sn}

To find the diffusivity $D$ and the exchange-current density $i_{0}$, we use the current evolution after a voltage step (see inset in Figure 2a) in PITT experiments on films of Sn. We use two different methods, the first using the Cottrell equation [35] and the second based on the paper by Li et al. [36]. The Cottrell solution gives the short-time $\left(D t / H^{2} \ll 1\right)$ current response following a step change in voltage starting from a uniform initial state assuming that the rate-limiting process is diffusion [36]:

$$
I(t)=\frac{Q}{H} \sqrt{\frac{D}{\pi t}},
$$




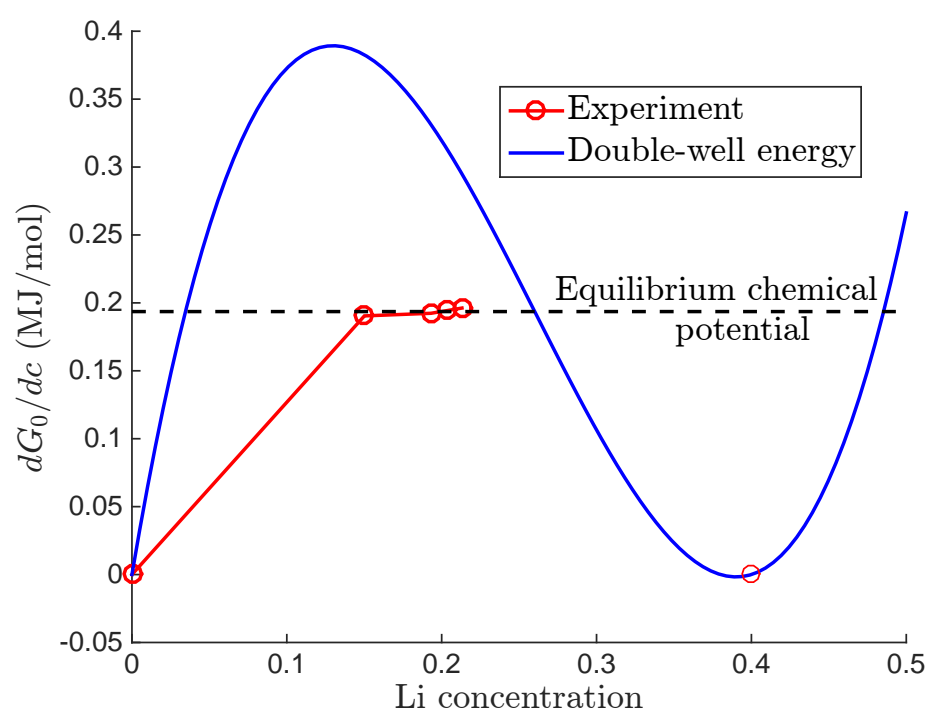

Figure 8: Homogeneous free-energy derivative from steady-state voltage vs charge measurements (Table 2) and using the double-well free-energy (Equations 2 and 36). The double-well predicts a nucleation potential about twice that found in experiment. Outside of nucleation however, the predictions match the experiments well since these are determined by the freeenergy near the equilibrium concentrations (close to $c=0.0346$ and $c=0.4853$ ).

where $I(t)$ is the current, $Q$ is the total charge transferred in the voltage step, $H$ is the film thickness, and $D$ is the diffusivity. Thus, this gives us only the diffusivity. Li et al. [36] derive analytical solutions for current evolution considering a finite surface-reaction rate. The short-time $\left(D t / H^{2} \ll 1\right)$ solution is given by

$$
I(t)=\frac{D Q B}{H} \exp \left(B^{2} D t\right) \operatorname{Erfc}(B \sqrt{D t})
$$

where $B=-i_{0}\left(d^{2} G_{0} / d c^{2}\right) /\left(\rho_{\mathrm{Sn}} F D R T\right)$. From this, we can determine both the diffusivity and the Butler-Volmer parameter $i_{0}$. When the surface-reaction is much faster than diffusion, $B \gg 1$, Equation 38 reduces to Equation 37. Equations 37 and 38 are used to fit the current evolution after a voltage step during PITT. Figure 9 shows one typical fit for each method. The kinetic parameter $M_{0}$ is found from the diffusivity using $D=M_{0}\left(d^{2} G_{0} / d c^{2}\right)$ with the free-energy curvature calculated from the steady state voltage vs charge measurements (Table 2 ). Table 3 shows values of the diffusivity, $M_{0}, i_{0}$ from such fits at different voltages (for the Sn phase).

\begin{tabular}{|c|c|c|c|c|c|}
\hline & \multicolumn{2}{|c|}{ Li et al. based [36] } & \multicolumn{2}{c|}{ Cottrell based } \\
\hline $\begin{array}{c}\text { Voltage } \\
\mathrm{V}\end{array}$ & $\begin{array}{c}i_{0} \\
\mu \mathrm{A} \mathrm{cm}\end{array}$ & $\begin{array}{c}M_{0} \\
10^{-21} \mathrm{~cm}^{2} \mathrm{sec}^{-1}\left(\mathrm{~J} \mathrm{~mol}^{-1}\right)^{-1}\end{array}$ & $\begin{array}{c}\text { Diffusivity } \\
10^{-16} \mathrm{~cm}^{2} \mathrm{sec}^{-1}\end{array}$ & $\begin{array}{c}\text { Diffusivity } \\
10^{-16} \mathrm{~cm}^{2} \mathrm{sec}^{-} 1\end{array}$ & $\begin{array}{c}\text { Slope in } \\
\text { log-log plot }\end{array}$ \\
\hline 0.72 & 0.29 & 0.76 & 1.50 & 1.56 & -0.46 \\
\hline 0.74 & 0.27 & 0.47 & 0.91 & 0.78 & -0.49 \\
\hline 0.76 & 0.41 & 0.14 & 0.06 & 0.05 & -0.50 \\
\hline
\end{tabular}

Table 3: Diffusivity, $M_{0}$ and $i_{0}$ of Sn using PITT (Figure 9). The two different fitting methods give similar values for diffusivity. The slope of the log-log plot being close to -0.5 suggests lithiation is diffusion limited.

Based on the Cottrell equation 37, the slope in a log-log plot of current vs time must be -0.5. This is the case in Table 3. The Cottrell solution assumes that the limiting process is diffusion. Table 3 thus suggests that for the Sn phase (Table 3), diffusion is rate-limiting. 


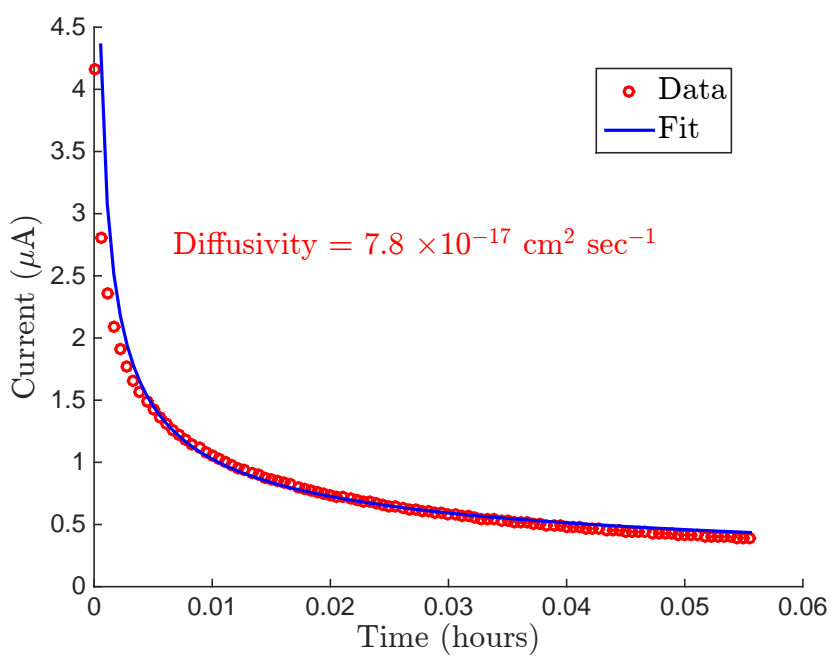

(a)

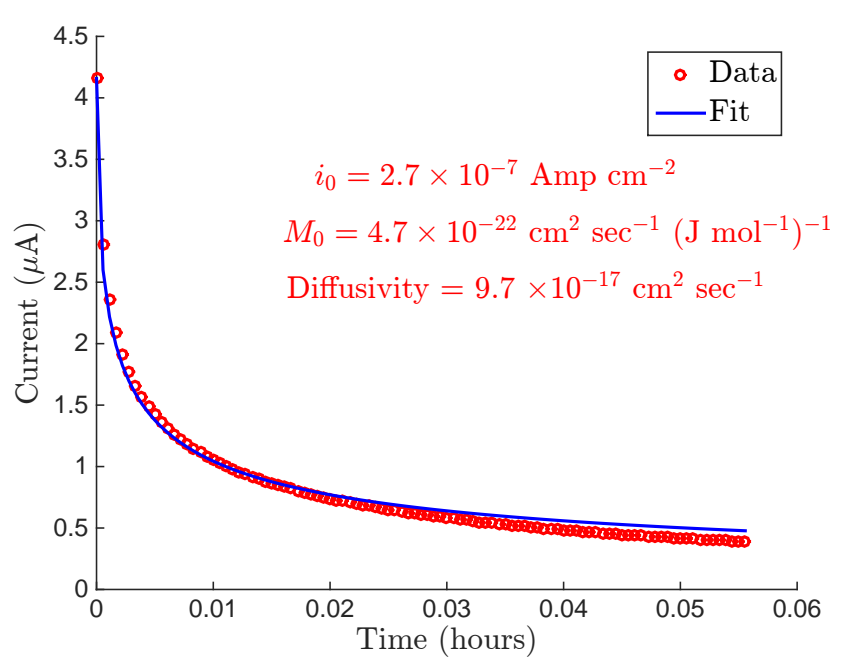

(b)

Figure 9: Current vs time following a voltage step in PITT experiments. Fits based on (a) Cottrell equation (b) Li et al. [36]. Results of such fits done at different voltages are tabulated in Table 3.

\section{3 $M_{0}, i_{0}$ for $\mathbf{L i}_{2} \mathbf{S n}_{5}$ and Interface mobility $\chi$}

To determine $M_{0}, i_{0}$ for $\mathrm{Li}_{2} \mathrm{Sn}_{5}$ and $\chi$ for the $\mathrm{Sn}_{-} \mathrm{Li}_{2} \mathrm{Sn}_{5}$ interface, we use galvanostatic and potentiostatic lithiation experiments. During galvanostatic lithiation, from Equation 33,

$$
\frac{d \bar{V}}{d \bar{x}_{\mathrm{int}}}=\frac{\bar{I}}{\bar{M}_{0}} .
$$

We performed an experiment in which we nucleated the $\mathrm{Li}_{2} \mathrm{Sn}_{5}$ phase and grew it to approximately half the film thickness, let it reach equilibrium, and lithiated/delithiated at constant current. Figure 10 shows the voltage evolution as a function of the interface position. The interface position is calculated from the total charge assuming the Li concentrations in the $\mathrm{Sn}$ and $\mathrm{Li}_{2} \mathrm{Sn}_{5}$ phases are equal to their equilibrium concentrations. From the slope of the linear fits and Equation 39, $M_{0}=9 \times 10^{-19} \mathrm{~cm}^{2} \mathrm{sec}^{-1}\left(\mathrm{~J} \mathrm{~mol}^{-1}\right)^{-1}$.

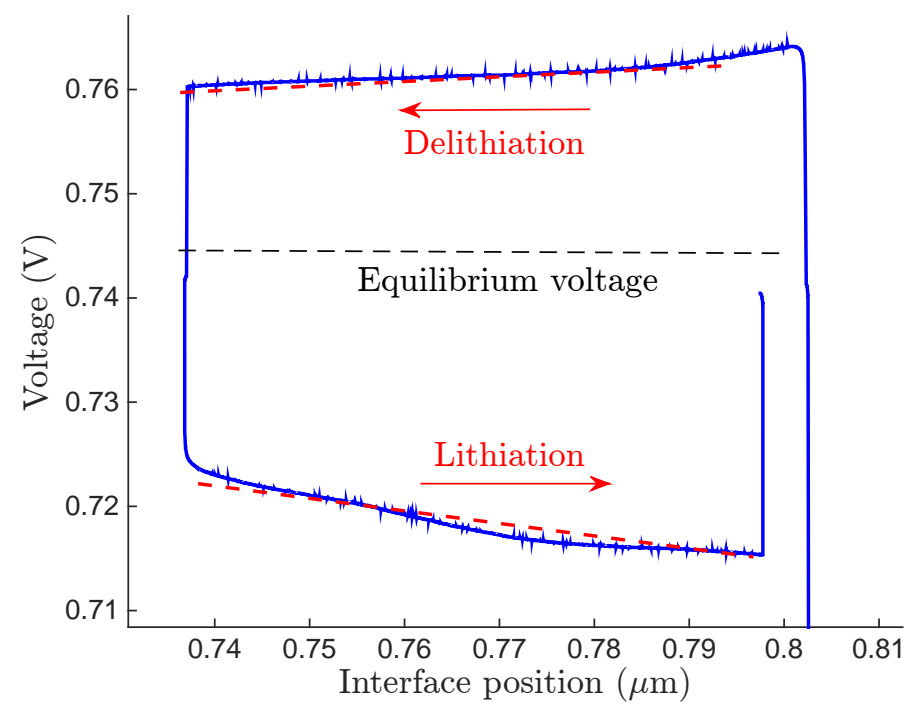

Figure 10: Evolution of voltage with interface position during galvanostatic lithiation/delithiation starting from an interface at equilibrium (blue). The slope of the line (the linear approximation is shown red) is used to determine $M_{0}$ using Equation 39. 
The determine $i_{0}$ and $\chi$, we repeat the galvanostatic lithiation experiments at different currents from $\mathrm{C} / 2500$ to $\mathrm{C} / 625$ (Figure 11a). The plateau voltage varies approximately linearly with the current (Figure 11b). From Equation (33), the slope of this line is given by,

$$
\text { Slope }=\frac{\bar{x}_{\text {int }}}{\bar{M}_{0}}+\frac{\bar{\chi}}{3 \bar{M}_{0}}+\frac{1}{\bar{i}_{0}} \text {. }
$$

The effects of $i_{0}$ and $\chi$ are indistinguishable in the galvanostatic and potentiostatic experiments (see Equations 33 and 40). Thus, from the slope and knowing $M_{0}$ and $x_{\text {int }}$ (estimated from the total charge), we can determine the possible ranges for $i_{0}$ and $\chi$ by setting $\chi=0$ and $i_{0} \rightarrow \infty$ respectively (Table 4 ).

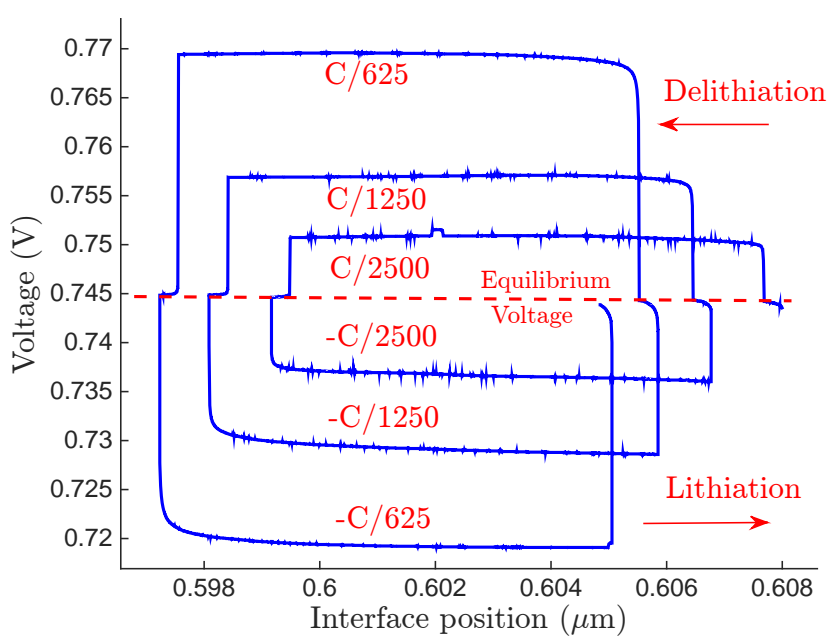

(a)

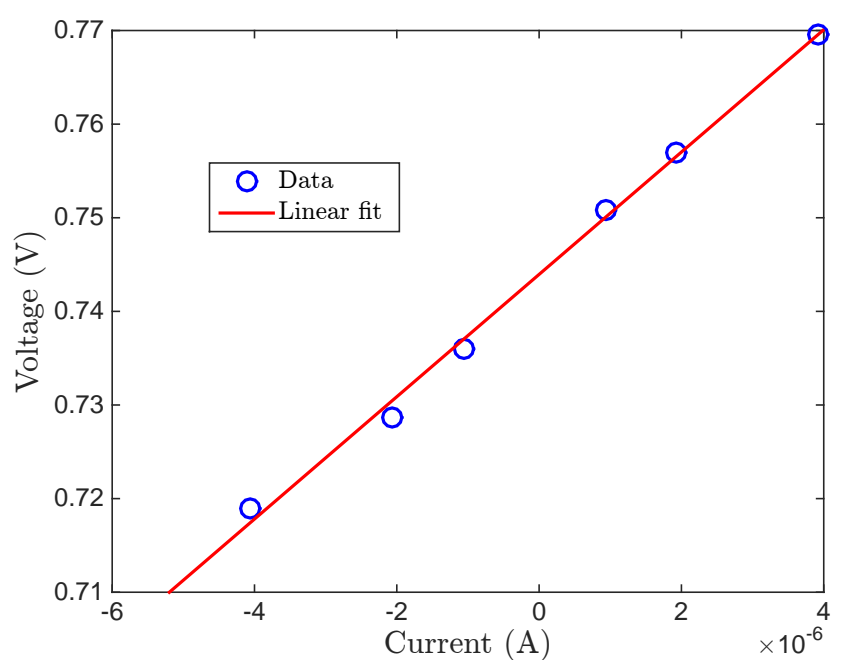

(b)

Figure 11: (a) Galvanostatic lithiation/delithiation at different currents starting from an interface at equilibrium. (b) The plateau potential in (a) varies approximately linearly with the current. The slope of this line is used to determine the ranges for $i_{0}, \chi$ (Table 4 ).

\begin{tabular}{|c|c|c|}
\hline$M_{0}\left(\mathrm{~cm}^{2} \mathrm{sec}^{-} 1\left(\mathrm{~J} \mathrm{~mol}^{-1}\right)^{-1}\right)$ & Minimum $i_{0}\left(\mu \mathrm{A} \mathrm{cm} \mathrm{cm}^{-2}\right)$ & $\operatorname{Max} \chi(\mu \mathrm{m})$ \\
\hline $9 \times 10^{-19}$ & 49.8 & 0.40 \\
\hline
\end{tabular}

Table 4: $M_{0}, i_{0}$, for $\mathrm{Li}_{2} \mathrm{Sn}_{5}$ and $\chi$ determined from galvanostatic experiments (Figure 11).

To determine $i_{0}$, we compare experiments and simulations of potentiostatic lithiation starting from an interface at equilibrium. The peak current following a voltage jump depends only on $i_{0}$ (Figure 12). From this, we get $i_{0} \approx 60 \mu \mathrm{A} \mathrm{cm}^{-2}$. Using this $i_{0}$, we get $\chi$ to be $0.07 \mu \mathrm{m}$ (using Equation (40)). 


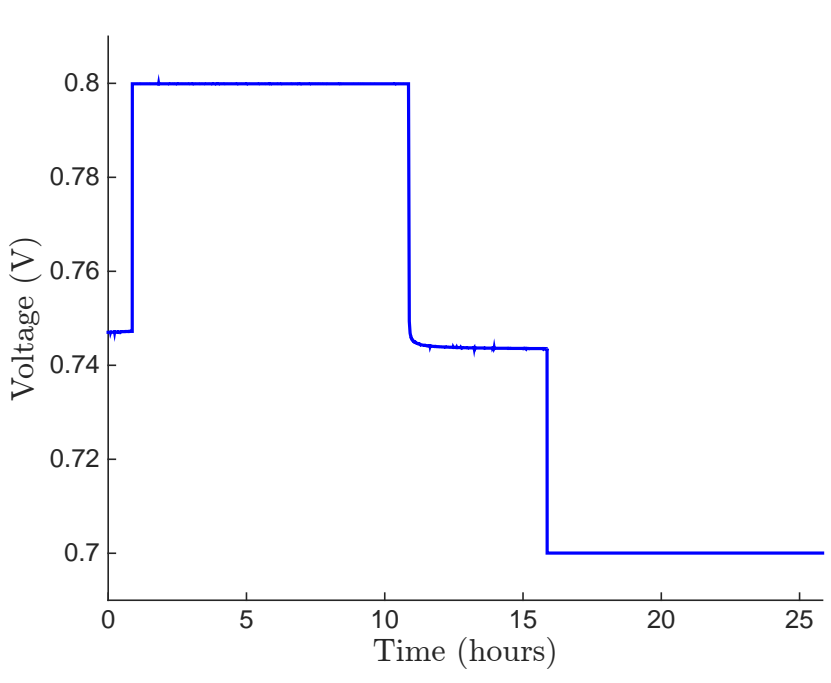

(a)

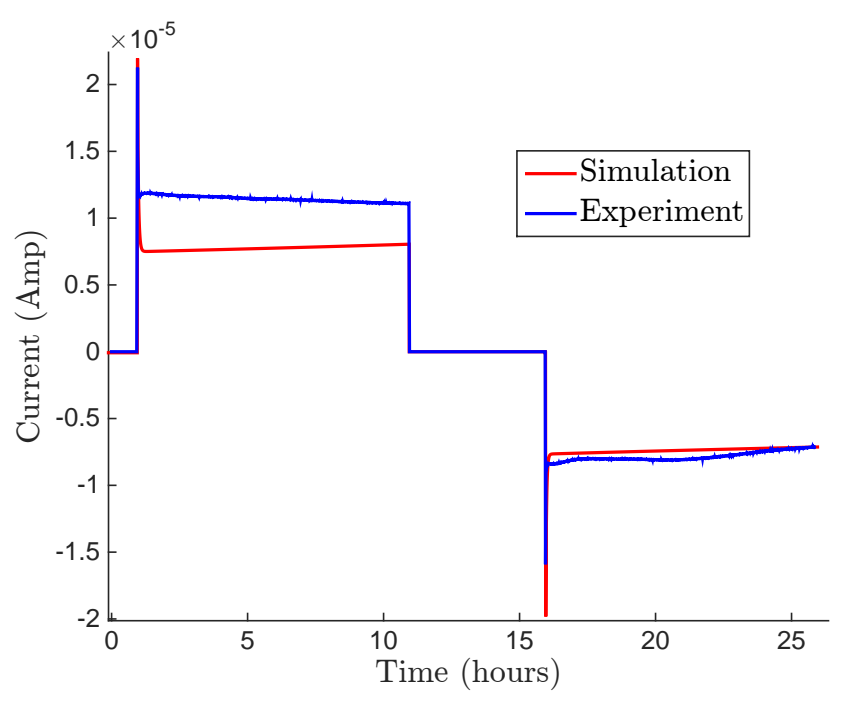

(b)

Figure 12: Starting with an interface at equilibrium, voltage steps are applied to lithiate/delithiate the film. (a) Applied voltage vs time and (b) corresponding current evolution in experiment (blue) and simulation (red). The peak current following a voltage step from equilibrium depends only on $i_{0}$. The peak current following the first voltage step is used to determine $i_{0}$ to be $60 \mu \mathrm{A} \mathrm{cm} \mathrm{cm}^{-2}$.

\subsection{Simulation with calibrated parameters}

\begin{tabular}{|c|c|}
\hline Parameter & Value \\
\hline Free-energy parameters & $\begin{array}{c}c_{0}^{\alpha}=0.0346, c_{0}^{\beta}=0.4853, \mu^{\mathrm{eq}}=0.193 \mathrm{MJ} \mathrm{mol}^{-1}, W=4.57 \mathrm{MJ} \mathrm{mol}^{-1} . \\
\text { (Equation 2) }\end{array}$ \\
\hline$M_{0}$ for $\mathrm{Sn}$ & $\begin{array}{c}0.14-0.76 \times 10^{-21} \mathrm{~cm}^{2} \mathrm{sec}^{-1}\left(\mathrm{~J} \mathrm{~mol}^{-1}\right)^{-1} \\
(\text { Equation } 3)\end{array}$ \\
\hline$D$ for $\mathrm{Sn}$ & $0.05-1.56 \times 10^{-16} \mathrm{~cm}^{2} \mathrm{sec}^{-} 1$ \\
\hline$i_{0}$ for $\mathrm{Sn}$ & $\begin{array}{l}0.29-0.41 \mu \mathrm{A} \mathrm{cm}^{-2} \\
\quad(\text { Equation } 4)\end{array}$ \\
\hline$M_{0}$ for $\mathrm{Li}_{2} \mathrm{Sn}_{5}$ & $9 \times 10^{-19} \mathrm{~cm}^{2} \mathrm{sec}^{-} 1\left(\mathrm{~J} \mathrm{~mol}^{-1}\right)^{-1}$ \\
\hline$D$ for $\mathrm{Li}_{2} \mathrm{Sn}_{5}$ & $4 \times 10^{-12} \mathrm{~cm}^{2} \mathrm{sec}^{-1}$ \\
\hline$i_{0}$ for $\mathrm{Li}_{2} \mathrm{Sn}_{5}$ & $60 \mu \mathrm{A} \mathrm{cm}^{-2}$ \\
\hline$\chi$ for $\mathrm{Sn}-\mathrm{Li}_{2} \mathrm{Sn}_{5}$ interface & $\begin{array}{c}0.07 \mu \mathrm{m} \\
\text { (Equation 3) }\end{array}$ \\
\hline
\end{tabular}

Table 5: Parameters in the Cahn-Hilliard model calibrated using experiments for the Sn $\leftrightarrow$ $\mathrm{Li}_{2} \mathrm{Sn}_{5}$ transformations.

Table 5 shows all the parameters in the Cahn-Hilliard model calibrated using experiments for $\mathrm{Sn} \leftrightarrow \mathrm{Li}_{2} \mathrm{Sn}_{5}$ transformations. Using these parameters, we compare simulations of the CahnHilliard equations with experiments.

Starting with an interface at equilibrium, we lithiate and delithiate galvanostatically at different currents allowing the film to go back to equilibrium between successive steps. The applied current is shown in Figure 13a and the resulting voltage, in both experiment and simulation, is shown in Figure 13b. The deviations of the voltage from equilibrium in the simulations show good agreement with experiments. 


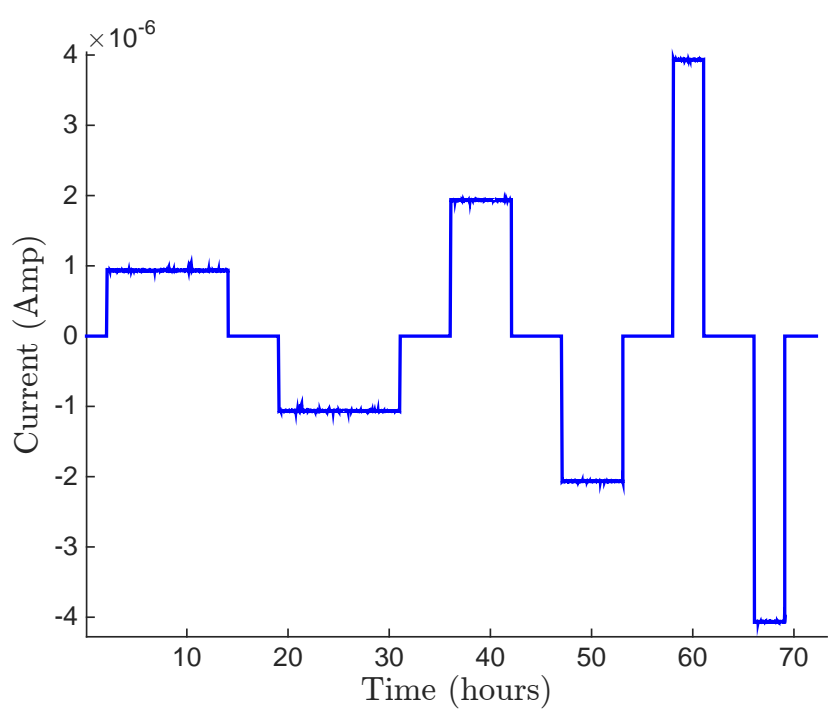

(a)

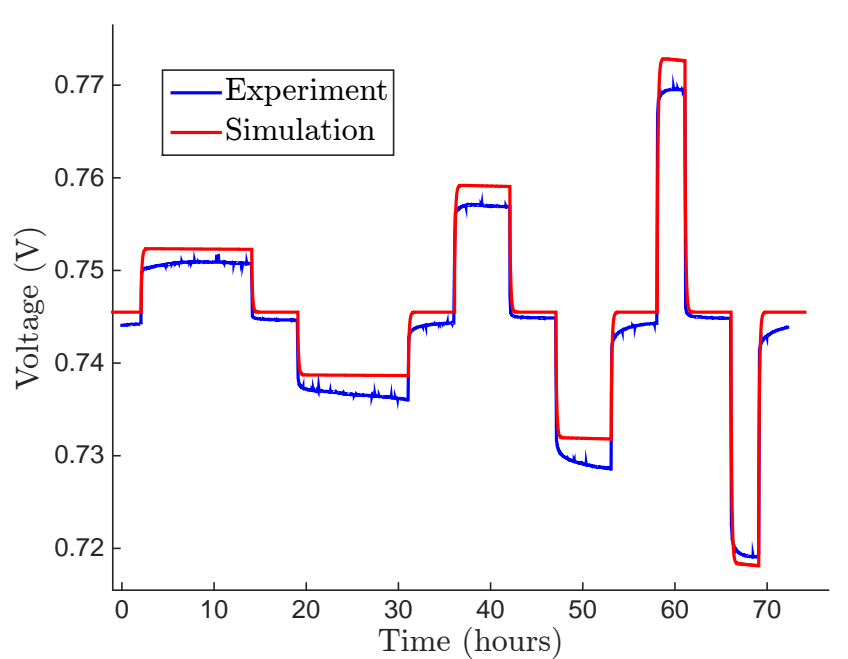

(b)

Figure 13: Starting with an interface at equilibrium, we lithiate and delithiate galvanostatically at different currents allowing the film to go back to equilibrium between successive steps. (a) Applied current vs time and (b) the resulting voltage evolution in experiment and simulation.

Figure 14 compares potentiostatic lithiation experiments and simulations of the same at three voltages, $0.64 \mathrm{~V}, 0.65 \mathrm{~V}$, and $0.665 \mathrm{~V}$. In the experiment, the film was initially in the $\mathrm{Sn}$ phase. On lowering the voltage, the $\mathrm{Li}_{2} \mathrm{Sn}_{5}$ phase nucleated and propagated into the film. In the simulations, we started with two existing phases with the interface close to the boundary (since the nucleation potential based on the double-well free-energy is about twice that in experiment. See Figure 8). The simulations underpredict the peak current following the voltage step due to the fact that a new phase is not nucleated in simulations. Figure 14b shows one of the experiments $(0.665 \mathrm{~V})$. The subsequent decay of the current in the model closely matches the experiment.

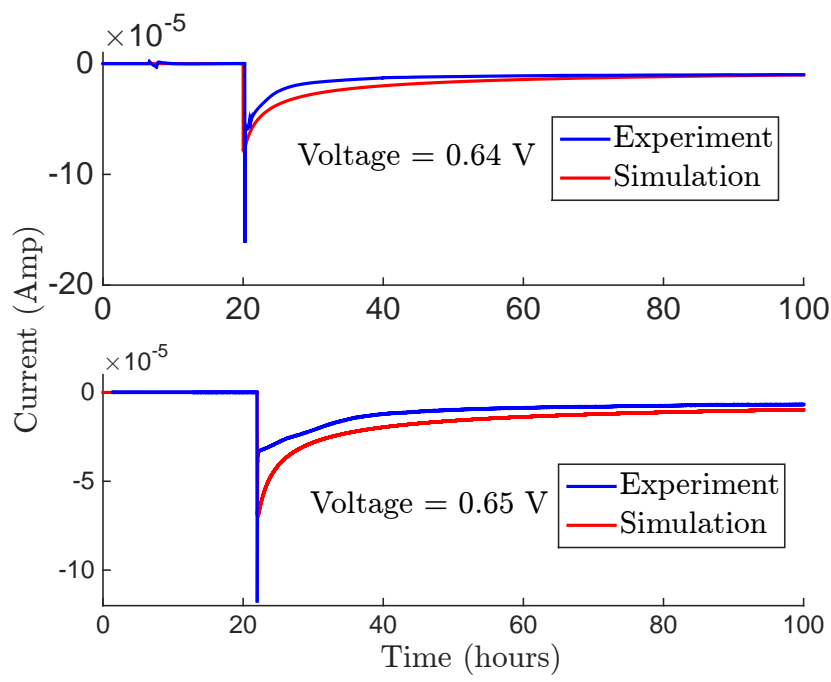

(a)

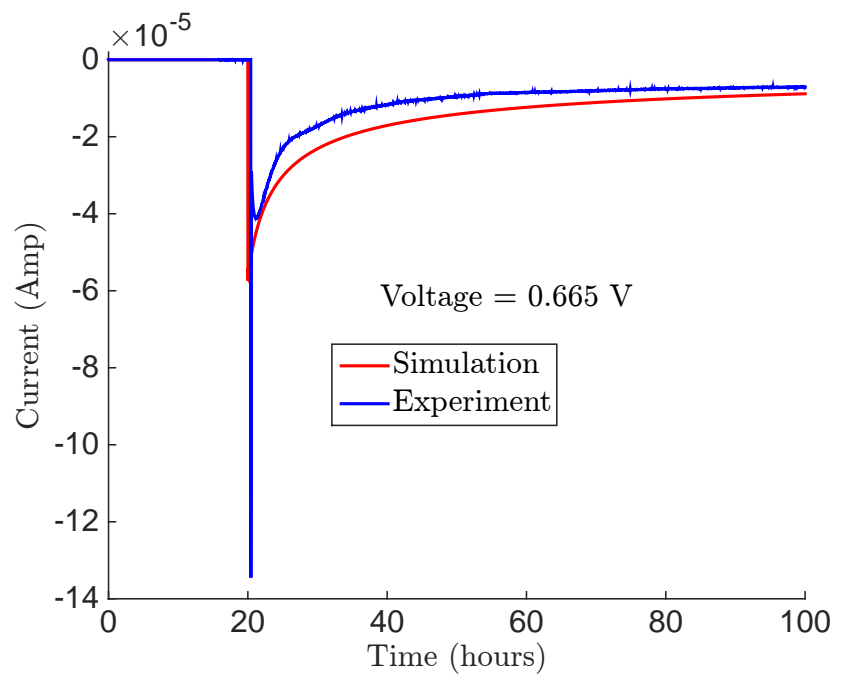

(b)

Figure 14: Comparison of current evolution in potentiostatic experiments and simulations three different voltages. The simulations underpredict the peak current following the voltage step due to the fact that a new phase is not nucleated in simulations while the subsequent decay closely matches the experiment. 


\section{Discussion}

In this paper, we have calibrated a modified Cahn-Hilliard model for Li-Sn electrodes that experience a transformation from $\mathrm{Sn}$ to $\mathrm{Li}_{2} \mathrm{Sn}_{5}$. Although Cahn-Hilliard models have been used widely in studying battery electrodes and have been very helpful in understanding qualitative features of microstructure evolution, not many attempts have been made to make quantitative predictions. For example, our calibration of the quartic double-well free-energy (Equation 2) shows that it is impossible to predict correctly the stoichiometric concentration of the $\mathrm{Sn}$ and $\mathrm{Li}_{2} \mathrm{Sn}_{5}$ phases, the plateau potential, and the nucleation potential for the $\mathrm{Sn} \rightarrow \mathrm{Li}_{2} \mathrm{Sn}_{5}$ transformation (Section 6). We expect the other commonly used free-energy model with the logarithmic free-energy of mixing to have similar shortcomings. This calls for a more flexible way of including experimentally determined free-energy into Cahn-Hilliard simulations.

We highlight the significance of interface mobility with the example of $\mathrm{Sn}-\mathrm{Li}_{2} \mathrm{Sn}_{5}$ and show that standard Cahn-Hilliard equations result in infinite interface mobility. One way of including a finite interface mobility within the Cahn-Hilliard framework is to use a concentration-gradient dependent Li mobility $M$. The numerical solution of the modified Cahn-Hilliard equations requires only minor changes in codes for the standard Cahn-Hilliard model (see Appendix). The perturbation analysis (Section 4.2) is very insightful in understanding the nonequilibrium interface behavior and in calibration of interface constants.

The connection we made in Section 4 between a general sharp-interface model and the sharp limit of our modified Cahn-Hilliard model should be a guide in deriving more general Cahn-Hilliard models. The two nonequilibrium processes at the interface characterized by the constants $K_{1}$ and $K_{2}$ (Equation 13) correspond to different processes. $K_{1}$ relates to the flux of Li through an interface at rest while $K_{2}$ is related to interface motion, transforming material ahead of the interface from one phase to another. In the modified Cahn-Hilliard model presented here, the two are related as $K_{2}=3 K_{1}$. In general, we do not expect the two to be related in this way.

In the Cahn-Hilliard modeling of Li-ion battery electrode materials, not much attention has been paid to the role of interface behavior. The modified Cahn-Hilliard and its generalizations along with careful experiments should be useful in a wide range of scenarios where interface mobility is important, both within and beyond the study of battery electrodes.

\subsection{Implications for battery design}

\section{Rate-limiting process}

The three timescales in the modified Cahn-Hilliard model corresponding to bulk diffusion, the interface response, and the surface-reaction are given by:

$$
\tau_{\text {diff }}=\frac{H^{2}}{M_{0} W}, \quad \tau_{\text {int }}=\frac{\chi^{2}}{M_{0} W}, \quad \tau_{\text {surf }} \propto \frac{1}{i_{0}} .
$$

Depending on the values of the parameters for a particular system, we can determine the rate-limiting step during the charge/discharge process. Equating the diffusion and interface timescales, we get

$$
\tau_{\text {diff }}=\tau_{\text {int }} \Longrightarrow H=\chi .
$$

Thus, for films around and smaller than the size $\chi$, the interface behavior plays an important role in determining the lithiation/delithiation response. Thus, in the design of electrodes of these sizes, one must give interface mobility a careful consideration. Finite interface mobility is likely to be important in other electrode materials that undergo phase transformations. 


\section{Interfacial dissipation}

Apart from the dynamics of the charge/discharge process, interfacial mobility can be important in deciding the energy efficiency of the battery. Interface propagation is a dissipative process and the energy lost subtracts from the useful energy stored in the battery. For simplicity, let us consider the case when the electrode is being charged/discharged at a constant flux $J$. Further, assume that the current is small enough that diffusion is at steady state. In this case, the energy dissipation rates due to diffusion and interface propagation are given by Equation (18) with $j=J$ for $x$ in 0 to $a$ and 0 otherwise and Equation (16) with $j^{-}=J, j^{+}=0$. Using these,

$$
\dot{U}_{\mathrm{int}}=-\frac{A J^{2}}{2}\left(\frac{1}{K_{1}}+\frac{1}{K_{2}}\right), \dot{U}_{\mathrm{bulk}}=-\frac{A J^{2} a}{M_{0}} .
$$

In charging/discharging the electrode completely, the interface moves between $x=0$ and $x=H$. The velocity of the interface is given by $v=J / \Delta c_{0}$. Integrating the above dissipation rate, the total energy dissipated is given by

$$
U_{\mathrm{int}}=\frac{A J H \Delta c_{0}}{2}\left(\frac{1}{K_{1}}+\frac{1}{K_{2}}\right), U_{\mathrm{bulk}}=\frac{A J H^{2} \Delta c_{0}}{2 M_{0}} .
$$

Equating the two, the interfacial dissipation is as significant as the bulk one when

$$
H=M_{0}\left(\frac{1}{K_{1}}+\frac{1}{K_{2}}\right)
$$

For the modified Cahn-Hilliard case, this becomes $H=\chi / 3$ (see Equation 23). For systems with characteristic length scale larger than this, bulk dissipation is important while for smaller systems, the interfacial dissipation is dominant.

\section{Conclusions}

In this paper, we used experiments, numerical simulations, and analytical calculations to calibrate a modified Cahn-Hilliard model for Li-Sn thin film electrodes. PITT, Potentiostatic, and Galvanostatic experiments were conducted on Sn thin films measuring transient current and voltage evolution. A modified one-dimensional Cahn-Hilliard equation along with the ButlerVolmer equation for the insertion reaction was used to model the experiments. Comparing model predictions and experiments, we determined the equilibrium and kinetic properties of the $\mathrm{Sn}$ and $\mathrm{Li}_{2} \mathrm{Sn}_{5}$ phases and their phase boundary. The main conclusions of this study are:

- The standard Cahn-Hilliard model captures the nucleation of phases and diffusion of Li but results in infinite mobility of the phase boundary (Section 5).

- A concentration-gradient dependent kinetic parameter can be used to give the interface a finite mobility and model interface-limited processes. Perturbation analysis of CahnHilliard equations reveals that the kinetic relations implied by them are a particular special case of a more general class. This is useful in developing more general CahnHilliard models that properly capture the interface behavior (Sections 4 and 5).

- Analytical double-well free-energies such as Equation 2 though helpful for analysis and understanding qualitative behavior, can be significantly different from that determined by experiments (Figure 8). Predictive phase-field models require a better way of incorporating free-energy determined from experiments. 
- $M_{0}$ of $\mathrm{Sn}$ and $\mathrm{Li}_{2} \mathrm{Sn}_{5}$ differ by about 3 orders of magnitude which suggests that $M_{0}$ is concentration dependent. The diffusivity of $\mathrm{Li}$ in $\mathrm{Sn}$ is around $10^{-16} \mathrm{~cm}^{2} \mathrm{sec}^{-1}$ and in $\mathrm{Li}_{2} \mathrm{Sn}_{5}$ is around $10^{-12} \mathrm{~cm}^{2} \mathrm{sec}^{-} 1$ (Tables 3 and 5 ). For $\mathrm{Sn}$, this is slightly smaller than that reported in [28]. We know of no diffusivity measurements in $\mathrm{Li}_{2} \mathrm{Sn}_{5}$.

- The exchange current density $i_{0}$ for $\mathrm{Sn}$ is of the order $10^{-7} \mathrm{~A} \mathrm{~cm}^{-2}$ (Table 3) and for $\mathrm{Li}_{2} \mathrm{Sn}_{5}$ is of the order $10^{-5} \mathrm{~A} \mathrm{~cm}^{-2}$ (Table 4). As far as we know, this is the first measurement of $i_{0}$ for Li-Sn. This suggests that for nanometer scale films, insertion reaction will be rate limiting compared to diffusion (for the $\mathrm{Li}_{2} \mathrm{Sn}_{5}$ phase).

- The interface-mobility parameter $\chi$ (Equation 3) for the $\mathrm{Sn}_{-} \mathrm{Li}_{2} \mathrm{Sn}_{5}$ interface is $0.07 \mu \mathrm{m}$ (Table 5). This is important since in electrodes at length-scales of $\chi$, the interface behavior is rate limiting and contributes significantly to the total energy hysteresis (Section 7.1). This calls a more accurate method of measuring the interface mobility and possibly other ways of characterizing the nonequilibrium interface behavior.

\section{Acknowledgement}

This work was supported by the U.S. Department of Energy through DOE EPSCoR Implementation Grant no. DE-SC0007074.

\section{Appendices}

\section{A Finite elements for the modified Cahn-Hilliard equa- tions}

Here we give a brief summary of the finite element method used in solving the Cahn-Hilliard equations. We can express the governing equations (Equations 1) in weak form as

$$
\begin{aligned}
& \int_{0}^{H} \mu \delta \mu=\int_{0}^{H} \frac{d G_{0}}{d c} \delta \mu+\kappa \int_{0}^{H} \frac{d c}{d x} \frac{d \delta \mu}{d x} \\
& \int_{0}^{H} \frac{\partial c}{\partial t} \delta c=-\int_{0}^{H} M \frac{d \mu}{d x} \frac{d \delta c}{d x}+J \delta c(0) .
\end{aligned}
$$

Some of the boundary terms vanish because of the boundary conditions. Introducing interpolation functions $\mu=N^{a} \mu^{a}$ and $c=\bar{N}^{a} c^{a}$, and adopting a semi-implicit time integration scheme we obtain the following nonlinear equation system for $\mu^{a}, \Delta c^{a}$.

$$
\left[\begin{array}{l}
R_{a}^{\mu} \\
R_{a}^{c}
\end{array}\right]=\left[\begin{array}{c}
\int_{0}^{H}\left[\left(\mu+\Delta \mu-\frac{d G_{0}(c+\Delta c)}{d c}\right) N^{a}-\kappa \frac{d(c+\Delta c)}{d x} \frac{d N^{a}}{d x}\right] \\
\int_{0}^{H}\left[\frac{\Delta c}{\Delta t} \bar{N}^{a}+\frac{M_{0}}{1+\frac{\chi}{\Delta c_{0}}\left|\frac{d c}{d x}\right|} \frac{d(\mu+\theta \Delta \mu)}{d x} \frac{d \bar{N}^{a}}{d x}\right]
\end{array}\right]+\left[\begin{array}{c}
0 \\
-J \bar{N}^{a}(0)
\end{array}\right]
$$

where $0<\theta<1$. We have used $\theta=0.75$ in all our simulations. The free-energy term is nonlinear, so these equations must be solved using Newton-Raphson iteration. The linear equations for the corrections $d \mu^{b}, d c^{b}$ have the form

$$
\left[\begin{array}{cc}
K_{a b}^{\mu \mu} & K_{a b}^{\mu c} \\
K_{a b}^{c \mu} & K_{a b}^{c c}
\end{array}\right]\left[\begin{array}{c}
d \mu^{b} \\
d c^{c}
\end{array}\right]=-\left[\begin{array}{c}
R_{a}^{\mu} \\
R_{a}^{c}
\end{array}\right]
$$


The element stiffness and residual can be expressed in compact form by writing

$$
\left[\begin{array}{c}
\mu \\
d \mu / d x \\
c \\
d c / d x
\end{array}\right]=[B][\phi]
$$

where $[\phi]$ is the nodal degree of freedom vector and $[B]$ is the usual element interpolation matrix. The stiffness and residual can then be expressed as matrix operations

$$
[K]=[B]^{T}[D][B], \quad[R]=[B]^{T}[P]
$$

where

$$
[D]=\left[\begin{array}{cccc}
1 & 0 & -d^{2} G_{0} / d c^{2} & 0 \\
0 & 0 & 0 & -\kappa \\
0 & 0 & 1 / \Delta t & 0 \\
0 & \theta M & 0 & 0
\end{array}\right] \quad[P]=\left[\begin{array}{c}
\mu+\Delta \mu-d G_{0}(c+\Delta c) / d c \\
-\kappa d(c+\Delta c) / d x \\
\Delta c / \Delta t \\
M d(\mu+\theta \Delta \mu) / d x
\end{array}\right]
$$

Combining element degrees of freedom in the usual way yields a standard nonlinear finite element system of equations

$$
R(u)=F
$$

which are solved by Newton-Raphson iteration. The correction $\Delta u^{k}$ to the degree of freedom vector $u$ at the $k$ th iteration is obtained by solving the linear system

$$
K \Delta u^{k}=F-R\left(u^{-1}\right)
$$

where $K$ and $R$ are determined by assembling the element stiffness and residuals defined above.

The Butler-Volmer equation (Equation 4) can be included within the standard finite element framework by adding an additional node to the mesh, which has the voltage $\mathrm{V}$ as its degree of freedom; and adding an element to the mesh which connects the voltage node and the node at the surface of the mesh. The element has a generalized force vector

$$
[R]=\left[\begin{array}{c}
J(V, \mu) \\
0 \\
0
\end{array}\right]
$$

where $J$ is the flux calculated from the Butler-Volmer equation. The corresponding stiffness is

$$
[K]=\left[\begin{array}{ccc}
\partial J / \partial V & \partial J / \partial V & 0 \\
0 & 0 & 0 \\
0 & 0 & 0
\end{array}\right]
$$

\section{References}

[1] M. Tang, W. C. Carter, and Y.-M. Chiang. Annu. Rev. Mater. Res., 40, 501 (2010).

[2] M. T. McDowell, I. Ryu, S. W. Lee, C. Wang, W. D. Nix, and Y. Cui. Adv. Mater., 24, $6034(2012)$.

[3] P. Limthongkul, Y. I. Jang, N. J. Dudney, and Y.-M. Chiang. Acta Mater., 51, 1103 (2003).

[4] K. J. Rhodes, R. Meisner, M. Kirkham, N. Dudney, and C. Daniel. J. Electrochem. Soc., 159, A294 (2012). 2012. 
[5] V. A. Sethuraman, M. J. Chon, M. Shimshak, V. Srinivasan, and P. R. Guduru. J. Power Sources, 195, 5062 (2010).

[6] V. A. Sethuraman, V. Srinivasan, A. F. Bower, and P. R. Guduru. J. Electrochem. Soc., 157, A1253 (2010).

[7] J. Christensen and J. Newman. J. Solid State Electrochem., 10, 293 (2006).

[8] R. Deshpande, M. Verbrugge, Y.-T. Cheng, J. Wang, and P. Liu. J. Electrochem. Soc., 159, A1730 (2012).

[9] W. Dreyer, J. Jamnik, C. Guhlke, R. Huth, J. Moskon, and M. Gaberscek. Nat. Mater., 9, 448 (2010).

[10] W. J. Boettinger, J. A. Warren, C. Beckermann, and A. Karma. Annu. Rev. Mater. Res., $32,163(2002)$.

[11] L. Q. Chen. Annu. Rev. Mater. Res., 32, 113 (2002).

[12] B. C. Han, A. V. der Ven, D. Morgan, and G. Ceder. Electrochim. Acta, 49, 4691 (2004).

[13] D. A. Cogswell and M. Z. Bazant. ACS Nano, 6, 2215 (2012).

[14] D. Burch and M. Z. Bazant. Nano Lett., 9, 3795 (2009).

[15] G. K. Singh, G. Ceder, and M. Z. Bazant. Electrochim. Acta, 53, 7599 (2008).

[16] L. Anand. J. Mech. Phys. Solids, 60, 1983 (2012).

[17] J. W. Cahn and J. E. Hilliard. J. Chem. Phys., 28, 258 (1958).

[18] P. Bai, D. A. Cogswell, and M. Z. Bazant. Nano Lett., 11, 4890 (2011).

[19] M. Tang, H. Y. Huang, N. Meethong, Y. H. Kao, W. C. Carter, and Y.-M. Chiang. Chem. Mater., 21, 1557 (2009).

[20] C. V. Di Leo, E. Rejovitzky, and L. Anand. Int. J. Solids Struct., 67-68, 283 (2015). 2015.

[21] T. R. Ferguson and M. Z. Bazant. J. Electrochem. Soc., 159, A1967 (2012). 2012.

[22] C. J. Wen and R. A. Huggins. J. Electrochem. Soc., 128, 1181 (1981).

[23] J. Sangster and C. W. Bale. J. Phase Equilib., 19, 70 (1998).

[24] R. A. Huggins. J. Power Sources, 81-82, 13 (1999).

[25] M. Winter and J. O. Besenhard. Electrochim. Acta, 45, 31 (1999).

[26] C. J. Wen and R. A. Huggins. J. Solid State Chem., 35, 376 (1980).

[27] J. Wang, I. D. Raistrick, and R. A. Huggins. J. Electrochem. Soc., 133, 457 (1986).

[28] J. Xie, N. Imanishi, A. Hirano, Y. Takeda, O. Yamamoto, X. B. Zhao, and G. S. Cao. Solid State Ionics, 181, 1611 (2010).

[29] J. S. Langer and R. F. Sekerka. Acta Metall., 23, 1225 (1975).

[30] R. W. Balluffi, S. Allen, and W. C. Carter. Kinetics of materials. John Wiley \& Sons, 2005. 
[31] C. H. Chen, E. Chason, and P. R. Guduru. Measurement of the phase and stress evolution during initial lithiation of Sn electrodes. Unpublished.

[32] J. Newman and K. E. Thomas-Alyea. Electrochemical systems. John Wiley \& Sons, 2012.

[33] B. A. Boukamp, G. C. Lesh, and R. A. Huggins. J. Electrochem. Soc., 128, 725 (1981).

[34] G. Bucci, S. P. V. Nadimpalli, V. A. Sethuraman, A. F. Bower, and P. R. Guduru. J. Mech. Phys. Solids, 62, 276 (2014). 2014.

[35] A. J. Bard, L. R. Faulkner, J. Leddy, and C. G. Zoski. Electrochemical methods: fundamentals and applications, volume 2. Wiley New York, 1980.

[36] J. Li, X. Xiao, F. Yang, M. W. Verbrugge, and Y.-T. Cheng. J. Phys. Chem. C, 116, 1472 (2012). 